\title{
The Parameter-less Randomized Gravitational Clustering algorithm with online clusters' structure characterization
}

\author{
Jonatan Gomez • Elizabeth Leon • Olfa Nasraoui • \\ Fabian Giraldo
}

Received: 10 December 2012 / Accepted: 10 January 2014 / Published online: 3 May 2014

(C) Springer-Verlag Berlin Heidelberg 2014

\begin{abstract}
Although clustering is an unsupervised learning approach, most clustering algorithms require the setting of parameters (such as the number of clusters, minimum density or distance threshold) in advance to work properly. Moreover, discovering an appropriate set of clusters is a difficult task since clusters can have any shape, size and density and is harder in the presence of noise. In fact, the presence of noise can deteriorate the results of many of the clustering techniques that are based on the least squares estimate. This paper presents a data clustering algorithm that does not require a parameter setting process [the Parameter-less Randomized Gravitational Clustering algorithm (PL-RGC)] and combines it with a mechanism, based in micro-clusters ideas, for representing a cluster as a set of prototypes. In this way, a set of parameter estimation strategies, previously developed for the Randomized Gravitational Clustering (RGC), are combined with a newly developed stopping criterion, based on the average number of points merged iteration by iteration,
\end{abstract}

\footnotetext{
J. Gomez (凶)

Alife Research Group, Department of Computer Systems

Engineering, Universidad Nacional de Colombia,

Carrera 30 No. 45-03 , Bogotá, D.C., Colombia

e-mail: jgomezpe@unal.edu.co

E. Leon · F. Giraldo

Midas Research Group, Department of Computer Systems

Engineering, Universidad Nacional de Colombia,

Bogotá, D.C., Colombia

e-mail: eleonguz@unal.edu.co

F. Giraldo

e-mail: fagiraldo@unal.edu.co

\section{O. Nasraoui}

Knowledge Discovery and Web Mining Lab, Department

of Computer Engineering and Computer Sciences,

University of Louisville, Louisville, USA

e-mail: olfa.nasraoui@louisville.edu
}

to remove the parameter setting of the RGC algorithm. The performance of the proposed PL-RGC algorithm is evaluated experimentally on two types of synthetic data sets: data sets with Gaussian clusters and with non-parametric clusters and two types of real data sets: five classic machine learning classification data sets and one intrusion detection data set. Our results show that the proposed mechanism is able to deal with noise, finds the appropriated number of clusters and finds an appropriated set of cluster prototypes regardless the type of data is working on.

Keywords Data mining - Data clustering · Online clusters' characterization - Gravity base data analysis

\section{Introduction}

Clustering [1] is a descriptive unsupervised learning task, of data mining and exploratory data analysis, that aims at classifying unlabeled data points into different groups or clusters according to some similarity concept. Such groups division is done in such a way that members of the same group are as similar as possible, while members of different groups are as dissimilar as possible. Although clustering is considered an unsupervised learning approach, most of the clustering algorithms require the number of clusters $(k)$ in advance. Such is the case of partitional algorithms like $k$-means [2] and fuzzy $k$-means [3]. One way to find an appropriated number of clusters $(k)$, is by running the clustering algorithm using different values of $k$, and choosing the best value of $k$ according to some internal validation measure. However, this approach increases the computational cost considerably. Some algorithms, Density-based Clustering algorithms (DB-C), try to estimate $k$, based on estimations of data density and considering regions of high data density as candidate clusters, 
while the clustering process is carried on [4-6]. DBSCAN [7] is a DB-C algorithm used to discover arbitrarily shaped clusters in the presence of noise. Basically, a concept of data point neighborhood is defined as the set of points located at a distance lower or equal than a certain threshold (Epsilon). Then, points are categorized into core point (if they have at least a number of predefined neighbors (MinPoints)), border point if they have less than such predefined number of neighbors and belong to some neighborhood of a core point and noise point if they have fewer than such predefined number of neighbors and do not belong to some neighborhood of a core point. Then, a cluster is defined as a set of density-connected points that is maximal with respect to a concept of densityreachability. One difficulty in using DBScan is its sensitivity to the setting of the parameters Epsilon and MinPoints [8]. DenCLUE is a DB-C algorithm using Gaussian kernel density estimation [9]. Clusters are the local maxima of the estimated density function, and data points are assigned to the cluster using a hill-climbing procedure (points belonging to the same local maximum are assigned to the same cluster) that allows the estimation of the size of the clusters. MDE is another DB-C algorithm, which uses a robust statistical estimator in a modified objective function taking into account density and scale optimization [10].

Hierarchical Clustering algorithms (HC) find clusters by improving partitions of the data set in an iterative process $[11,12]$. Such partitions or clusters are improved by a series of successive merges or successive divisions. In this way, $\mathrm{HC}$ algorithms build a hierarchical structure, called dendogram, of the data (clusters) in an iterative process using one of two approaches: agglomerative or divisive. Agglomerative hierarchical clustering is a bottom-up approach. At the beginning, each data sample is a single cluster, then clusters are merged based on some similarity measure in an iterative process that stops when there is only one cluster remaining $[13,14]$. A divisive clustering approach is a top-down clustering approach that starts with all data samples belonging to one cluster, then splits this cluster recursively until each data sample forms its own cluster. Any desired number of clusters can be obtained by 'cutting' the dendogram at the proper level.

Chameleon is an agglomerative hierarchical clustering algorithm based on a dynamic model [8]. It is a two-phase algorithm that operates on a sparse graph of the data to be clustered (requires a similarity matrix), in which nodes represent data items and links represent the similarity among the data items. In the first phase, the algorithm does a partition of the graph into several small sub-clusters. In the second phase, Chameleon finds the final clusters by merging the subclusters in a hierarchical way. Chameleon uses the interconnectivity and closeness of the clusters for identifying the most similar pair of clusters, and for combining the sub-clusters in a hierarchical way. The interconnectivity and closeness are estimated by taking into account internal characteristics of the clusters, therefore when the clusters are merged the model can automatically adapt to the new internal characteristics of the merged cluster. Chameleon is able to find clusters of different shapes, densities, sizes, and can handle noise and artifacts. CURE (Cluster Using REpresentatives) [15] is an agglomerative $\mathrm{HC}$ algorithm that represents a cluster as a set of points that are initially selected to be scattered throughout the clusters, and then they get shrinked toward the center of the cluster. This representation allows the identification of clusters with non-spherical shape and is able to handle outliers. Balanced Iterative Reducing and Clustering using Hierarchies (BIRCH) [16] is a divisive $\mathrm{HC}$ algorithm which finds small clusters by scanning the data set only once. Then, these sub-clusters are clustered in memory using any clustering algorithm. BIRCH summarizes the whole data set in a special structure called Clustering Feature Tree (CF Tree). A $\mathrm{CF}$ tree is a height-balanced tree where each node is a Clustering Feature $(\mathrm{CF})$. A CF summarizes a sub-cluster using clustering information about its children nodes, such as centroid and covariance statistics.

As can be seen, clustering is a hard task since the number of 'true' clusters is unknown and such clusters can have any shape, size and density. Moreover, the clustering task is harder in the presence of noise, in fact, the presence of noise can deteriorate the results of many of the clustering techniques that are based on the least squares estimate [17]. Several approaches have tried to tackle this problem by introducing robust statistics [17,18], and by modifying the objective function being optimized to make the parameter estimation more robust with respect to noise [19-21]. Data clustering algorithms inspired by natural phenomena such as natural evolution $[6,22]$ and gravitational force have also been proposed [23-25].

In particular, Gravitational data Clustering algorithms (GC) are considered agglomerative $\mathrm{HC}$ algorithms based on concepts of field theory in physics $[23,24]$. Basically, the data clustering problem is solved by considering each data point as a particle ${ }^{1}$, with unitary mass, in a space exposed to gravitational fields (generated by such particles), then simulating the dynamics of this gravitational system following Newton's gravitational law, and finally extracting the hierarchy of emergent clusters of particles [23]. There are two main issues with Wright's GC algorithm: it has a high time complexity, $O\left(N^{3}\right)$, with respect to the size of the data set $(N)$ [24] and it is not able to deal with noise (every point, noisy or not, is assigned to a cluster).

Gomez et al. [25] proposed a new gravitational clustering algorithm in that is robust to noise and does not require the

\footnotetext{
${ }^{1}$ Notice that we talk about particle instead of data points, since, in general, particles are allowed to be defined as the fusion of several data points.
} 
number of clusters in advance. Like GC, it is inspired from the theory of gravitation, however it redefined the clustering target and the dynamics of the system, thus reducing the time complexity of the original GC to less than quadratic in addition to being able to resist noise. The computational complexity was reduced mainly by considering only a sample instead of all the other data points when making a decision about moving a given data point. Then, both interacting data points are moved according to a simplified version of the Universal Gravitational Law and Second Newton's Motion Law. Points that are close enough end up merged into virtual clusters. Finally, the big crunch effect (convergence to one single big cluster at the end) was eliminated by introducing a cooling mechanism similar to the one used in simulated annealing. The proposed clustering algorithm, called Randomized Gravitational Clustering (RGC) worked well on a variety of data sets. However, its performance was sensitive to the choice of three parameters: initial gravitational 'Constant' $(G)$, minimum cluster size $(\alpha)$, and maximum number of iterations $(M)$. Moreover, the RGC algorithm represents clusters using the set of points belonging to them and does not use a compact representation of clusters. Later, Gomez et al. in [26] generalized the RGC algorithm and eliminated the initial gravitational constant $(G)$ parameter by estimating it using an extended binary search method. Then, Gomez et al. in [27] eliminated the minimum cluster size parameter $(\alpha)$ of the RGC algorithm and introduced a refinement strategy for merging overlapping clusters. Finally, Leon et al. in [28] used the rich dynamic of the RGC algorithm and the concept of micro-cluster to represent a cluster as a set of prototypes reducing the amount of information required for representing a cluster.

In this paper, we present the Parameter-less Randomized Gravitational Clustering Algorithm (PL-RGC), a data clustering algorithm that does not require the setting of parameters, and combine it with the mechanism for obtaining an online (while the RGC is running) clusters structure characterization of the data set using Micro-clusters. Basically, we define an stopping criterion, removing the necessity of setting a maximum number of iterations $(M)$, by analyzing the number of new clustered points the RGC algorithm is producing (iteration by iteration). Then, we combine this estimation with our previously proposed Initial Gravitational constant $(G)$ estimation [26], our minimum cluster size parameter estimation $(\alpha)$ [27] and our micro-cluster characterization [28]. We perform several different experiments on synthetic and real data sets, and the obtained results are reported and analyzed. This paper is divided into seven sections. Section 2 outlines the gravitational law physics concepts required for developing the gravitational clustering algorithm and the classic Gravitational Clustering algorithm. Section 3 describes the Randomized Gravitational Clustering algorithm. Section 4 introduces the set of methods for estimating the parameters of the RGC algorithm including the newly proposed stopping criterion, presents the proposed Parameter-less Randomized Clustering Algorithm (PL-RGC), and explains the strategy for generating online prototypes. Section 5 shows some experiments performed on synthetic data sets, while Sect. 6 shows some experiments performed on real data sets. Finally, Sect. 7 draws some conclusions and our future work.

\section{Gravitational clustering}

\subsection{Newton's law of gravity}

In Newton's gravitational law $[29,30]$, each particle $x$ exerts an attractive force on any other particle $y$. This force is exerted along the direction joining particles $x$ and $y$, directly proportional to the product of the particle masses, and inversely proportional to the square of the particles distance, see Eq. 1.

$F_{x, y}=\frac{G m_{x} m_{y}}{d_{x, y}^{2}}$

Here, $m_{x}$ and $m_{y}$ are the masses of particles $x$ and $y$, respectively, $d_{x, y}$ is the Euclidean distance between the two particles, and $G$ is a gravitational constant. Notice that, particle $y$ is exposed to the gravitational field of particle $x$, that pulls $y$ toward the center of this field, with intensity $F_{x, y}$ and direction $\overrightarrow{d_{x, y}}=\vec{x}-\vec{y}$. Therefore, the gravitational force vector $\overrightarrow{F_{x, y}}$ is defined as:

$\overrightarrow{F_{x, y}}=\frac{G m_{x} m_{y}}{\left\|\overrightarrow{d_{x, y}}\right\|^{2}} \overrightarrow{e_{x, y}}$

where, $\overrightarrow{e_{x, y}}$ is the unitary vector with direction $\overrightarrow{d_{x, y}}$. Since, $\overrightarrow{e_{x, y}}=\frac{\overrightarrow{d_{x, y}}}{\left\|\overrightarrow{d_{x, y}}\right\|}$, Eq. 2 can be rewritten as:

$\overrightarrow{F_{x, y}}=\frac{G m_{x} m_{y}}{\left\|\overrightarrow{d_{x, y}}\right\|^{3}} \overrightarrow{d_{x, y}}$

Finally, the force vector $\vec{F}_{y}$ applied on $y$ due to the gravitational fields of the other particles in the system, is defined as:

$\overrightarrow{F_{y}}=\sum_{x=1}^{N} \overrightarrow{F_{x, y}}=\sum_{x=1}^{N} \frac{G m_{x} m_{y}}{\left\|\overrightarrow{d_{x, y}}\right\|^{3}} \overrightarrow{d_{x, y}}$

\subsection{Force field simulation}

The movement of a particle in a force field can be simulated by using Newton's laws of movement, and considering the 
acceleration induced by the force field on that particle [29]. Moreover, a simplified and less precise movement simulation can assume that the particle's instant ${ }^{2}$ velocity is zero and that the particle's instant acceleration $(\vec{a})$ is constant. In this way, the position of a particle $y$ can be approximated using Eq. 5 .

$y_{t+\Delta(t)}=y_{t}+\frac{\vec{a} \Delta(t)^{2}}{2}$

Therefore, the next position of a particle $y$ due to the gravitational influence of another particle $x$ can be approximated by Eq. 6, while the next position of a particle $y$ due to the complete force field (i.e., the field force of all the other particles) can be approximated using Eq. 7.

$$
\begin{aligned}
& y_{t+1}=y_{t}+\frac{G m_{x} m_{y}}{\left\|\overrightarrow{d_{x, y}}\right\|^{3}} \overrightarrow{d_{x, y}} \\
& y_{t+1}=y_{t}+\sum_{x=1}^{N} \frac{G m_{x} m_{y}}{\left\|\overrightarrow{d_{x, y}}\right\|^{3}} \overrightarrow{d_{x, y}}
\end{aligned}
$$

\subsection{Clustering algorithm}

The gravitational clustering algorithm (GC) proposed by Wright in [23] is an agglomerative hierarchical clustering algorithm based on the concepts of field theory in physics [24], see Algorithm 1. The data clustering problem is solved by considering each data point as a particle ${ }^{3}$, with unitary mass (lines 1-3), in a space exposed to gravitational fields, then simulating the dynamics of this gravitational system following Newton's gravitational law (lines 5-8), and finally extracting the hierarchy of emergent clusters of particles [23] (lines 10-15). Following this line, Wright proposed the gravitational clustering algorithm by using Eq. 4 for defining the force vector applied to a particle and Eq. 7 for moving them (line 6). When two particles are close enough to be merged (line 11), they are considered to belong to the same cluster (line 12), one of them is removed from the simulation (lines 14 and 15), and the mass of the other is increased by the amount of the mass of the particle being removed (line 13). The process is stopped when only one particle remains in the system (line 4).

As can be noticed, Wright's GC algorithm has a high time complexity, $O\left(N^{3}\right)$, with respect to the size of the data set $(N)$ [24], making it not suitable for large data sets. Moreover, the gravitational clustering algorithm does not automatically detect the number of clusters nor is it able to deal with noise.

\footnotetext{
$\overline{2}$ Here instant means during a short interval of time $\Delta(t)$.

${ }^{3}$ Notice that we talk about particle instead of data points, since, in general, particles are allowed to be defined as the fusion of several data points.
}

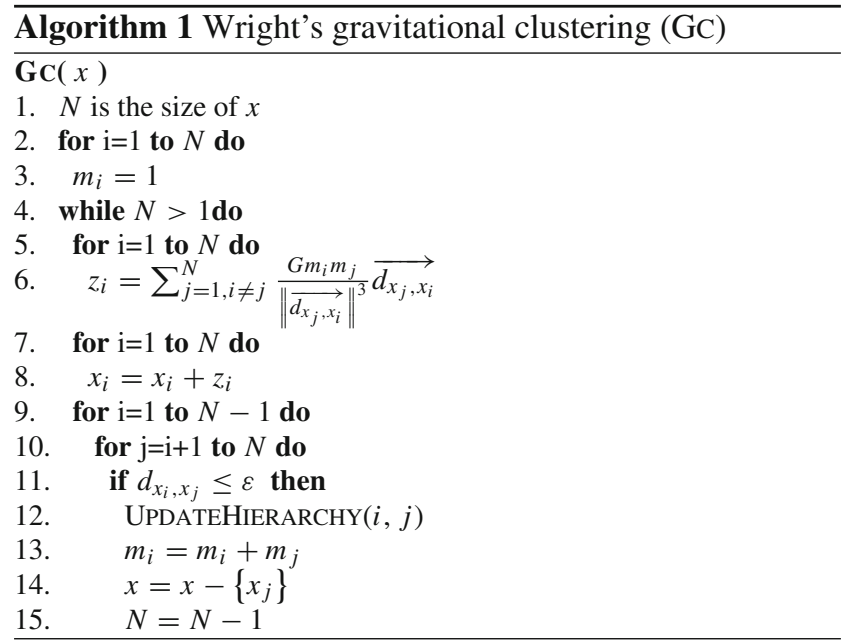

\section{Randomized Gravitational Clustering algorithm (RGC)}

Gomez et al. proposed a gravity-based clustering algorithm, called Randomized Gravitational Clustering algorithm (RGC), that reduces the time complexity of Wrights algorithm, is robust to noise and determines the number of clusters to be extracted in [25], see Algorithm 2. The computational complexity is reduced to super-linear $(O(n \sqrt{n}))$ by considering just another randomly selected point, instead of all the other data points, when moving a given point (lines 5 and 6) and by introducing a cooling mechanism. In this way, both interacting data points are moved according to a simplified version of the Universal Gravitational Law and Second Newton's Motion Law, see Eqs. 8 and 9.

$$
\begin{aligned}
& \overrightarrow{F_{x, y}}=\frac{G}{\left\|\overrightarrow{d_{x, y}}\right\|^{3}} \overrightarrow{d_{x, y}} \\
& y_{t+1}=y_{t}+\overrightarrow{d_{x, y}} \frac{G}{\left\|\overrightarrow{d_{x, y}}\right\|^{3}}
\end{aligned}
$$

Notice that each data point is always a particle in the gravitational system (no point is removed neither the mass is changed), and points that are close enough (line 7) end up merged into virtual clusters using a disjoint sets structure [31] (lines 2, 7 and 9). The big crunch effect is eliminated by introducing a cooling mechanism similar to the one used in simulated annealing (line 8). The gravitational system dynamics is simulated during a maximum number of iterations $M$ previously defined (line 3). Finally, an extra parameter $(\alpha)$ is used to determine the minimum number of data points that a cluster should include to be considered a valid cluster, see Algorithm 3. Clusters with size lower than $\alpha$ are considered noisy clusters. 

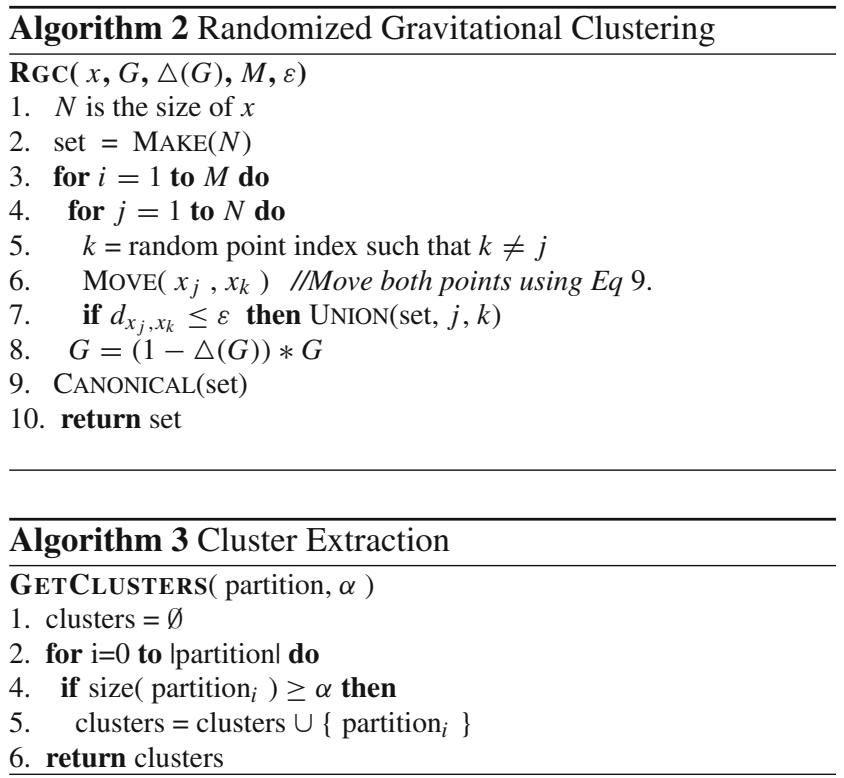

Gomez et al. in [26], introduced the notion of greatest minimal distance in the RGC algorithm to reduce the effect of the data set size in the system dynamics, i.e., when moving and merging points. In this way, the greatest minimal separation between points $(\hat{d})$, when uniformly separated, is estimated ${ }^{4}$ using:

$\widehat{d}=\frac{2 \sqrt{n}}{\sqrt{3} N^{\frac{1}{n}}}$

Here, $N$ is the number of data points in the $n$-dimensional $[0,1]$ Euclidean space. The two-dimensional hexagonal packing of circles approach [33] was used for computing this value, i.e., to estimate the greatest minimal distance between points, such points are considered as defining a grid defining isosceles triangles (pyramids). The height of such a triangle is $\frac{2}{\sqrt{3}}$ the side of the triangle, and the maximum number of points per dimension of such a grid should be bounded by $N^{\frac{1}{n}}$. We introduced a $\sqrt{n}$ correction factor for data sets where the number of points is considerably low compared to the number of vertices on the hypercube $[0,1]^{n}$, i.e., $\left(2^{n}\right)$. In this way, $\hat{d}$ is used as a renormalization factor in the gravitational force law, and both the vector gravitational force Eq. 8 and the movement Eq. 9 are rewritten as:

$\overrightarrow{F_{x, y}}=G \overrightarrow{d_{x, y}}\left(\frac{\widehat{d}}{\left\|\overrightarrow{d_{x, y}}\right\|}\right)^{3}$

\footnotetext{
$\overline{4}$ The problem of determining the optimal arrangement of points in such a way, the greatest minimal separation between points is obtained, is an open problem in Geometry [32].
}

$y_{t+1}=y_{t}+G \overrightarrow{d_{x, y}}\left(\frac{\widehat{d}}{\left\|\overrightarrow{d_{x, y}}\right\|}\right)^{3}$

Notice that, this gravitational force is acting as a contrast operator that gives more influence to closest points (points at a distance lower than $\hat{d}$ ) while giving less influence to farther points (points at a distance higher than $\hat{d}$ ).

\section{Parameter-less Randomized Gravitational Clustering (PL-RGC)}

As can be seen, the RGC algorithm requires the setting of five different parameters: initial gravitational constant $(G)$, cooling factor $(\triangle(G))$, merging distance $(\varepsilon)$, and maximum number of iterations $(M)$. As shown by Gomez et al. in [25], the performance of the RGC algorithm is not very sensible to the setting of the cooling factor and merging distance, and that good values for these two parameters are $\Delta(G)=0.01$ and $\varepsilon=0.0001$. Moreover, Gomez et al. in [26] defined an automatic estimation of the initial gravitational constant value $(G)$ for an appropriated performance of the RGC algorithm. In this section, we remove the maximum number of iterations parameter $(M)$ by adding a stopping criterion that automatically determines when the gravitational system dynamics should not be simulated anymore.

\subsection{Stopping criterion}

In order to determine when the RGC algorithm should stop, we can use the number of cluster fusions produced in every iteration. Due to the dynamic nature of the RGC algorithm, there is a moment in time when the number of cluster fusions will start to decrease as the time goes by. This behavior is expected, since the probability of merging two points belonging to the same cluster will increase as the cluster's size increases. Therefore, the probability of picking two points from different clusters will decrease in time. Let $f_{i}$ be the number of cluster fusions performed during iteration $i$. It is clear that a low value of $f_{i}$ will indicate some kind of stability in the cluster formation at iteration $i$. However, just considering the number of cluster fusions in one iteration is not enough for determining this stability tendency due to the random nature of the RGC algorithm. Taking this into consideration, we compute, at every iteration $i$, the average number of cluster fusions during the last 30 iterations, i.e., $\bar{f}_{i}=\frac{\sum_{k=0}^{29} f_{i-k}}{30}$. If $\bar{f}_{i}$ is less that 1 , then we stop the RGC algorithm at the end of iteration $i$. Notice that this value indicates that it is not expected to merge clusters or to include new points in the already defined clusters. This criterion is applied after 30 initial iterations. 


\subsection{Initial $G$ value}

An extended bisection search algorithm is used [31]. Basically, the number of clustered points [points that were assigned to some cluster with two or more points (CLUST PNTS)], after some checking iterations of the RGC algorithm, is used as an indicator of the quality of $G$. In this way, the value of $G$ is considered appropriate if the number of clustered points is close to $\frac{N}{2}$, i.e., $\frac{N}{2} \pm \tau$, after $\lfloor\sqrt{N}\rfloor$ iterations (these values were obtained after analyzing the behavior of the cluster formation when running the RGC algorithm on different data sets). If more than half of the points $\left(\frac{N}{2}\right)$ are clustered, then $G$ is reduced, otherwise $G$ is increased. Algorithm 4 calculates the initial value of $G$ using the bisection search. Notice that Algorithm 4 is a super-linear algorithm $O(N \sqrt{N})$ with respect to the data set size, since the number of iterations performed by the bisection search algorithm is, in general, constant with respect to the size of the data set.

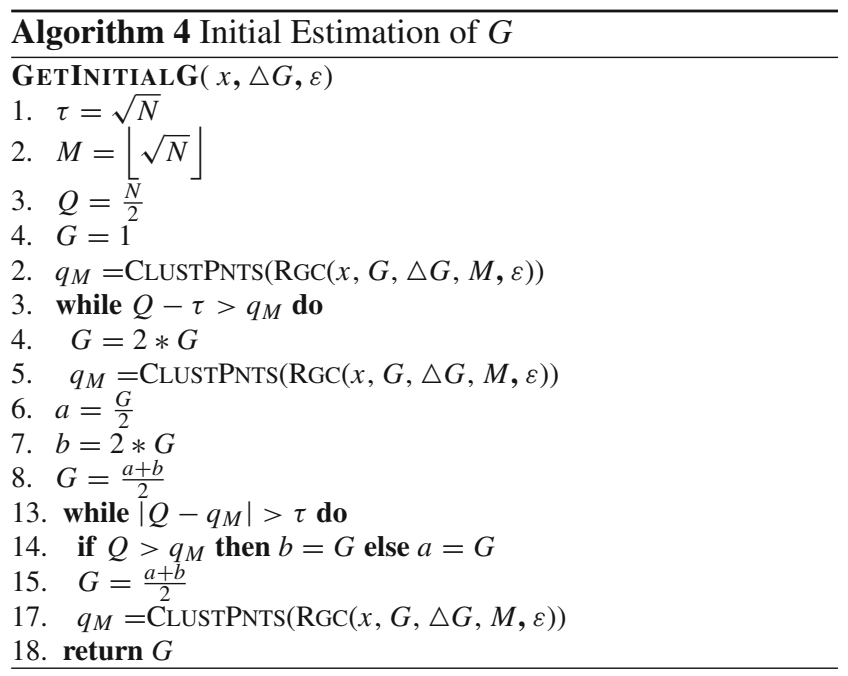

\subsection{Estimation of the minimum cluster size $(\alpha)$}

Gomez et al. in [27] estimated the minimum cluster size parameter $(\alpha)$ of the RGC algorithm, i.e., the number of data points that a cluster should contain to be considered a valid cluster, by analyzing the behavior of the number of clusters $\left(c_{m}\right)$ with at least $m$ points. First, noise points are expected to be far enough from any other point, thus reducing the possibility of being merged into clusters, therefore noisy points would be merged in clusters with a low number of points. Second, noise is expected to be present in different regions of the space, therefore it is expected that the number of clusters with a low number of points (noise points) will be high. Third, it is expected that real clusters will be defined by a large number of points. Fourth, it is expected, in general, that the number of clusters defining the data set is very small compared to the number of points represented by such clusters. Figure 1 shows the expected behavior of the number of clusters $c_{m}$ with at least $m$ points.

Therefore, we compute the behavior of $c_{m}$ and obtain the size $\alpha$ where the slope is closest to $\frac{\pi}{4}$, i.e., we choose the value $\alpha$ where the number of clusters $((n c)$ with at least $\alpha$ points is higher than the value $\alpha(n c>\alpha)$, see Fig. 1 .

\subsection{Improving the clusters quality}

Due to the random nature of the merging process of the RGC algorithm, it is possible that some good points, points that already belong to some cluster, will not be assigned to some cluster. Moreover, it is possible that some "overlapping" clusters will not be merged at all. In order to tackle these disadvantages, Gomez et al. in [27] introduced a notion of a data point's region of action based on the merging process, and we propose a heuristic for refining the extracted clusters based on this notion. Due to the dynamic behavior of the RGC, it is possible not only to track the cluster formation, but also to track the real distance (distance in the original space before points were moved) between points that have been merged. This distance gives us an indication of the strength of attraction force exerted by the region of action of the data point. In this way, we associate with every data point $k$, two values: the aggregated merging distance of data point $k$ (noted $d_{k}$ ) and the total number of data points that data point $k$ has being merged with (noted $n_{k}$ ). Notice that $d_{k}$ and $n_{k}$ can be computed in constant time, by incrementing $d_{k}$ and $n_{k}$ every time that a data point $j$ is merged with data point $k$ - just by adding to $d_{k}$ the original distance between data points $k$ and $j\left(d_{x_{k}, x_{j}}^{*}\right)$ and by adding one to $n_{k}$. Finally, we compute the average merging distance $\left(\overline{d_{k}}=\frac{d_{k}}{n_{k}}\right)$ of data point $k$, and we use it as the radius of the region of action (a hypersphere centered at the data point with radius $\overline{d_{k}}$ ) defined by data point $k$. We define the concept of a data point's region of action, having in mind two main objectives: The first one is to be able to determine the cluster that an unknown and potentially new data point should belong to, and the second one is to be able to refine clusters by merging "overlapping clusters". In this direction, we define the sub-cluster relation between clusters as follows:

One cluster $c_{1}$ is said to be a sub-cluster of a cluster $c_{2}$ if every data point $x$ of cluster $c_{1}$ falls inside the region of action of some data point $y$ in cluster $c_{2}$. As expected, two clusters will be merged if one of them is a sub-cluster of the other one. This process can be repeated until no further clusters are merged.

\subsection{Prototypes generation}

Because the RGC algorithm can be seen as a hierarchical and agglomerative algorithm, it is possible to use the con- 
Fig. 1 Expected relation between the number of cluster with at least $m$ points

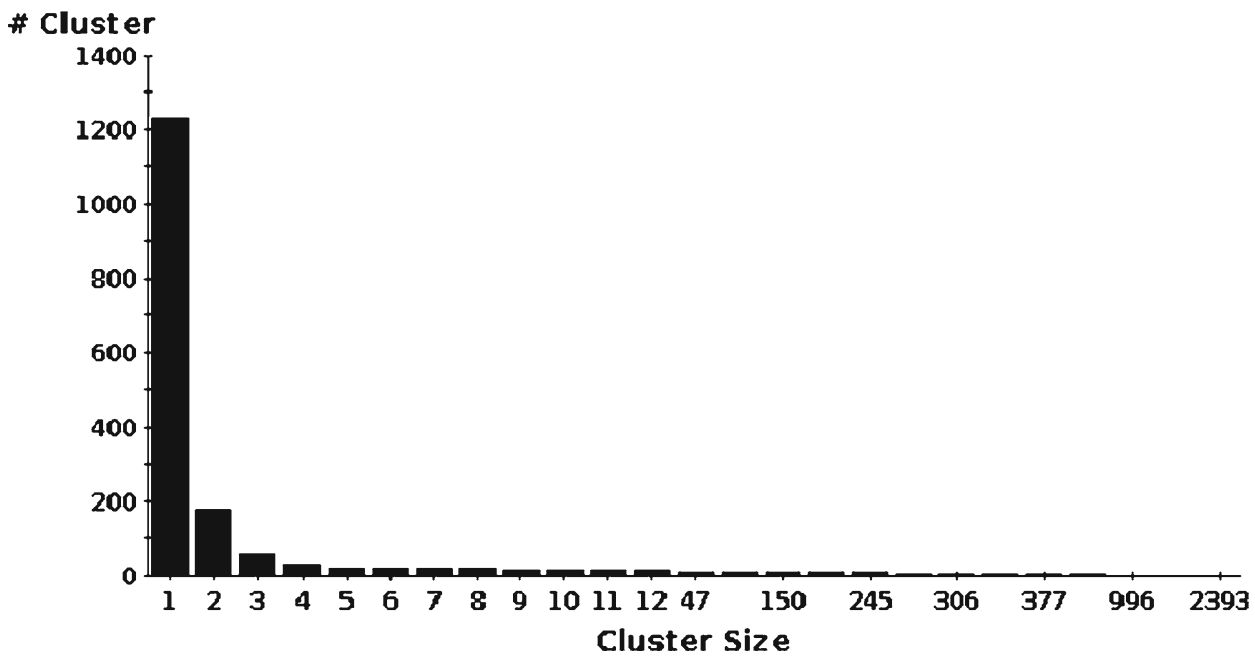

ceptual hierarchical structure created by RGC to summarize (online) the emergent clusters and sub-clusters being formed iteration through iteration as proposed by Leon et al. in [28]. In this way, an analysis and summarization process (PROTOTYPEGENERATION) is applied after certain number of iterations ( $I$ ) of the RGC algorithm. In this process (Algorithm 5), the union/disjoint set structure (labels structure) is analyzed to determine if new clusters' prototypes or high level clusters' prototypes should be introduced into the prototypes hierarchy structures (line 1). A cluster is considered as candidate prototype if such cluster contains a number of points higher than a certain predefined threshold $\delta$ and it has not been absorbed (merged) by other prototype (line 4). Therefore, we check the labels' structure to find clusters with size higher than the given threshold (line 4). Such new prototypes are added to the clusters' prototype hierarchical structure as leaves (line 6). Then, we check if old clusters are merged (clusters being re-labeled). If so, we introduce a high level cluster prototype (prototype defined by other cluster prototypes) as an inner node having as child nodes the cluster prototypes defining it, (lines 8-16). Notice that, clusters' prototypes are defined at the same time they are summarized, i.e., the average of the points defining the cluster is computed and stored in the cluster prototypes hierarchy along with the cluster size (line 13). A similar process is applied when two cluster prototypes are merged but taking into consideration the average and the size of the prototypes (additive property of the clusters' prototype). Finally, some cluster prototypes can absorb some points. Such points information is also used to recompute the summarization information of the cluster (line 4).

Figure 2 shows an illustrative example of the application of the clustering prototype generation mechanism. At iteration 1, two clusters are formed: point 1 and 2 are merged, and points 8 and 9 are merged. At iteration 2, point 3 is absorbed by cluster $\{1,2\}$; and point 10 is absorbed by cluster $\{8,9\}$.

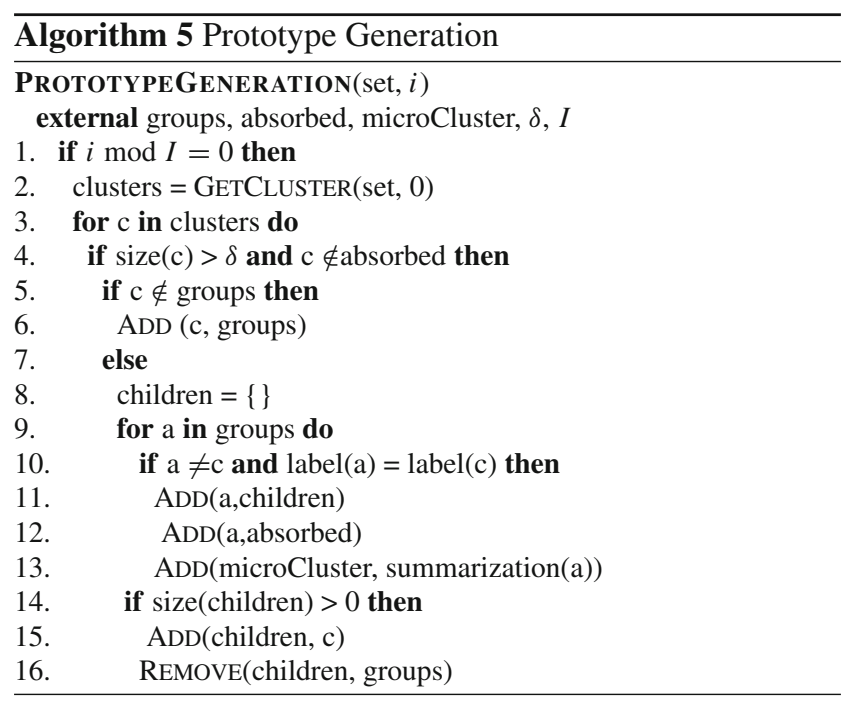

Since iteration 2 is a check point (summarization and analysis iteration) and the minimum cluster size (for defining a cluster prototype) is set to 3 , then two clusters' prototype are added in the clusters' prototype hierarchy $(\{1,2,3\}$ and $\{8,9,10\})$. When the checkpoint is reached again, a new cluster prototype $(\{5,6,7\})$ is formed and merged with cluster $\{1,2,3,4\}$. Notice that point 4 is absorbed by cluster $\{1,2,3\}$. Therefore, a new high-level cluster prototype is added to the clusters' prototype hierarchy.

\subsection{The PL-RGC algorithm}

Algorithm 6 presents the full version of the Parameter-less Randomized Gravitational Clustering (PL-RGC) algorithm with online clusters characterization using micro-clusters. The cooling factor $(\triangle G)$ and the merging distance $(\varepsilon)$ are set to the values indicated by Gomez et al. in [25] (lines 1 and 2 ), while the initial value for constant $G$ is estimated using 
Fig. 2 Example of prototype hierarchy definition

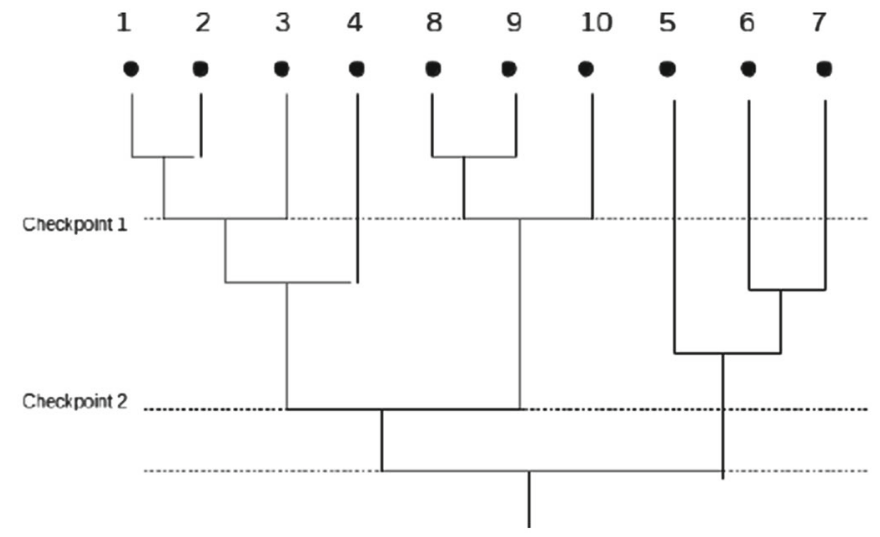

$\{1,2,3\} \quad\{8,9,10\}$

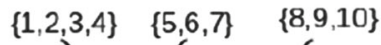

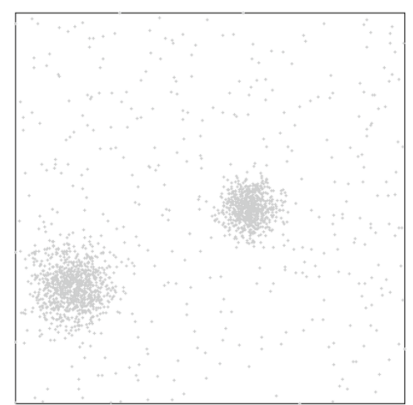
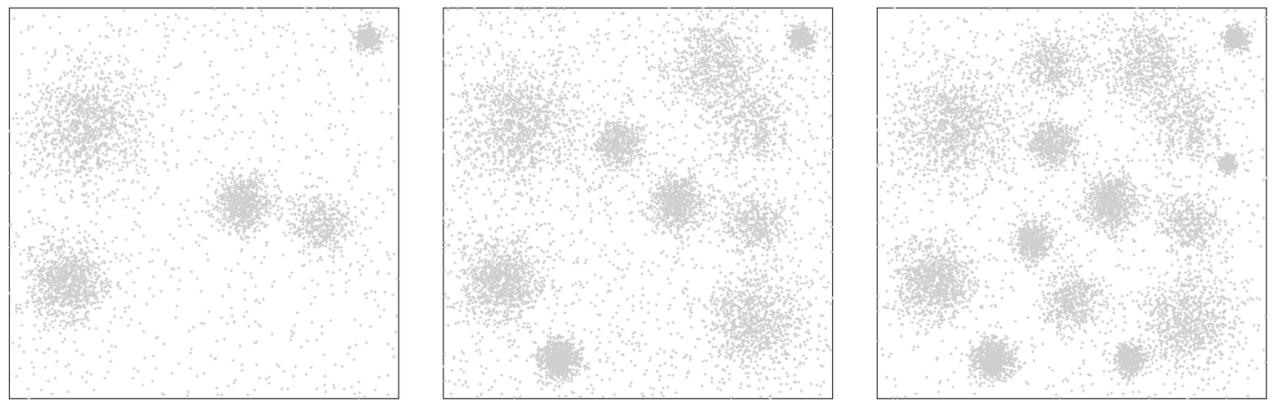

Fig. 3 Data sets with Gaussian clusters: a 2 clusters, b 5 clusters, c 10 clusters, and $\mathbf{d} 15$ clusters

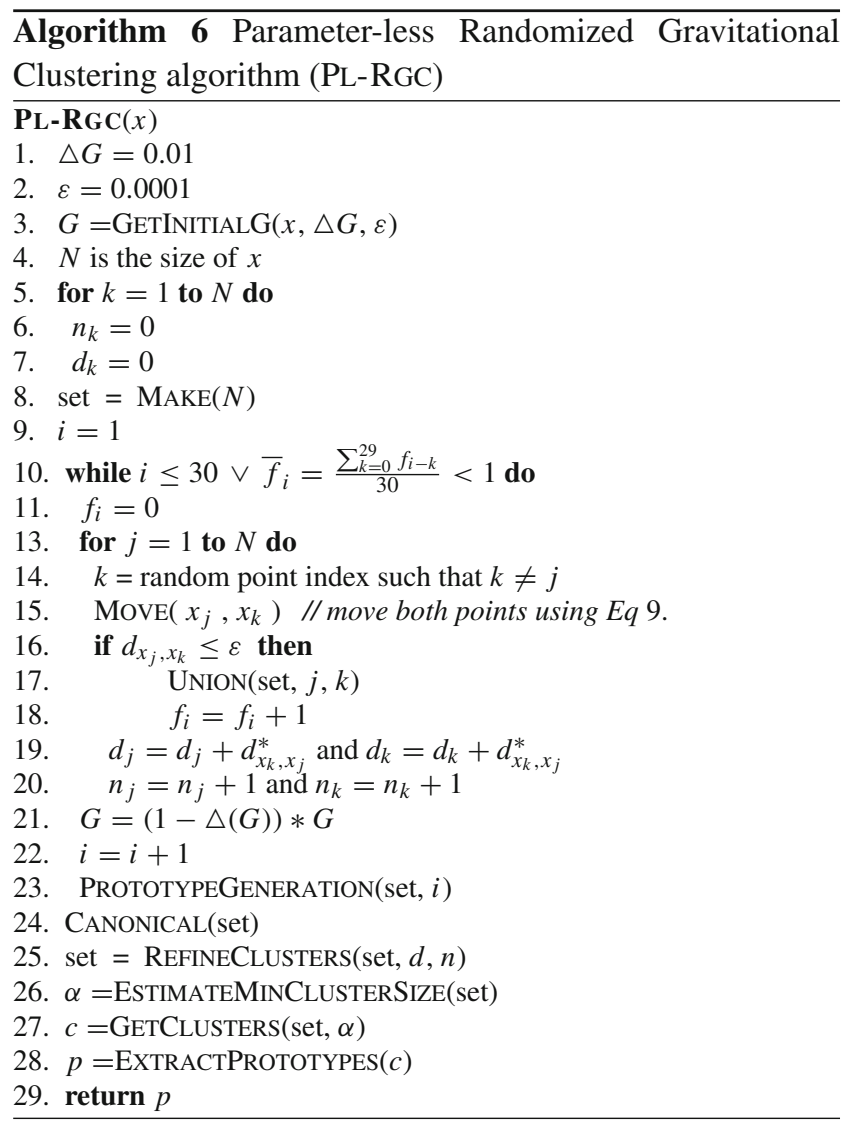

the procedure described in Sect. 4.2 (line 3). The PL-RGC is using the stopping criterion proposed in Sect. 4.1 (lines 10-11 and 18), the clusters refinement procedure, Sect. 4.4, proposed by Gomez et al. in [27] (lines 5-7, 19-20 and 25), the prototypes generation, Sect. 4.5 , proposed by Leon et al. in [28] (lines 23, 28 and 29), and the minimum cluster size $(\alpha)$, Sect. 4.3, proposed by Gomez et al. in [27] (lines 26-27). Notice that the PL-RGC does not produce a set of cluster but a set of prototypes for each one of the discovered clusters.

\section{Experiments with synthetic data sets}

\subsection{Gaussian clusters data sets}

Tests were conducted on four synthetic data sets with different clusters density and size, and with different percentages of noise (10 and $20 \%$ ), see Fig. 3. We set the minimum micro-cluster size to 10 , and we check the disjoint-set structure (looking for micro-clusters) every 20 iterations. We show the full set of results for the ten clusters data set with $20 \%$ noise, but the results obtained for other data sets are similar. Figure 4 shows the typical clustering result obtained every 250 iterations up to the end of the process (when the stopping criterion is applied). Notice that, the majority of data points inside the clusters are moving towards the cluster cen- 


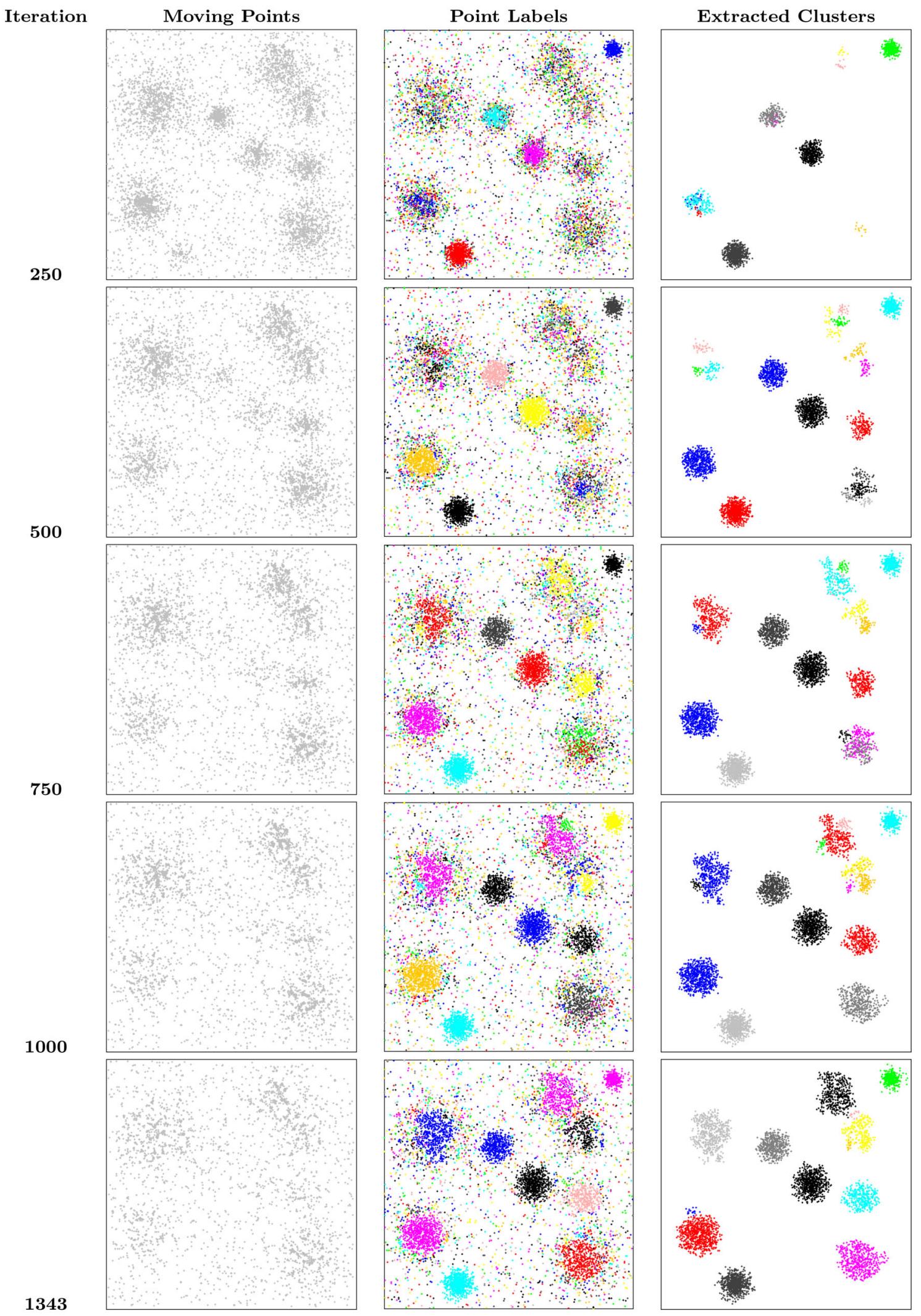

Fig. 4 Typical clustering result for the Gaussian ten clusters data set with $20 \%$ of noise every 250 iterations. Column one shows the position of the data points after the given number of iterations, column two shows the clusters obtained after the given number of iterations, and column three shows the extracted clusters after such number of iterations 
Fig. 5 Clusters refinement process on the ten Gaussian clusters data set. a Clusters obtained by the PL-RGC algorithm before refinement, $\mathbf{b}$ clusters obtained by the PL-RGC algorithm after refinement, $\mathbf{c}$ micro-clusters

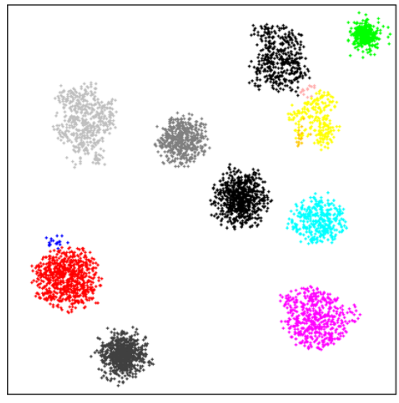

(a)

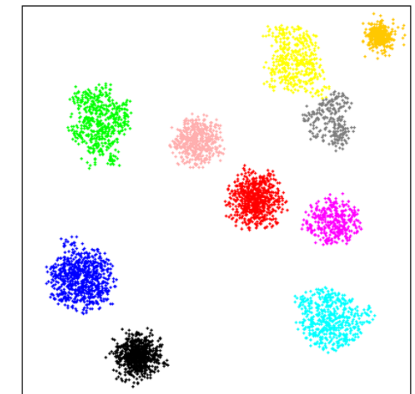

(b)

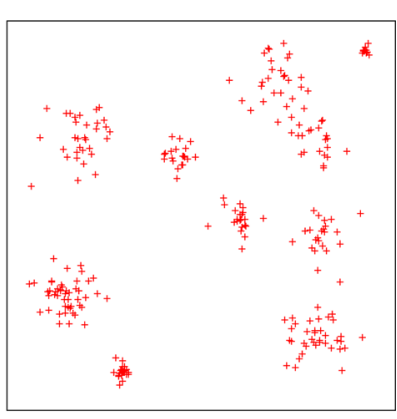

(c) ters (see first column), while almost most of the noisy points remain still or barely move. As expected, clusters emerge as the dynamic system is simulated; first, the most dense clusters (see point labels column at iteration 250) and then the less dense clusters (see point labels column at iteration 750). Moreover, noisy data points do not form clusters or they form very tiny clusters. It can be seen that, the proposed heuristic for determining the minimum size of valid clusters works well, since almost every noisy point is removed and only "real" clusters are extracted (see third column). Finally, it looks like both the simplification of the heuristic for estimating the initial gravitational constant $G$ and the heuristic for stopping the RGC algorithm work well, since, in general, no "real" clusters were merged and the structure of the extracted clusters does not change much between the last two shown iterations (see iteration 1,000 and 1,343) indicating that no further changes are expected.

Figure 5 shows the result of applying the refinement to the extracted clusters ${ }^{5}$ and the final set of prototypes (microclusters). As can be noticed, some small clusters were merged with bigger clusters producing a more compact cluster model. Moreover, for this data set, only 70 prototypes are obtained. These micro-clusters capture the structure of the original data set, determine appropriately the clusters in the data set and are just $1 \%$ of the original data set in size (from 6,400 data points to 70 representative points). As can be noticed, noisy points are removed and no representative points are generated for noise data points.

Figure 6 shows the behavior of the PL-RGC algorithm on all the four Gaussian clusters data sets. As expected, the behavior is similar for these data sets to that observed on the ten cluster data set. However, for the 15-cluster data set, RGC divided one of the clusters in a few smaller ones. It can be due to the fact that such a cluster is not dense and is located between two very dense cluster. Anyway, all the clusters are extracted, noise is removed and small number of prototypes (micro-clusters) are generated.

\footnotetext{
$\overline{5}$ It is possible to apply the refinement process to the generated clusters before extracting them. However, we just perform it after extracting the clusters for simplifying the analysis of the refinement process.
}

We evaluated the performance of PL-RGC against DBScan, Fig. 7 shows the performance reached by DBScan with different values of MinPoints and Epsilon. As can be seen, DBScan is able to find a good approximation of the clusters, if appropriate parameters are given. We say a good approximation because, in general, DBScan generates several small clusters around the big clusters that it found. Also, some clusters are merged and some noisy clusters are generated.

Figure 8 shows the performance reached by DBScan on all the four Gaussian clusters data sets using different MinPoint values and 0.14 as epsilon parameter. We fixed the parameter epsilon to 0.14 after trial and error because it showed the best behavior. As expected, DBScan finds good cluster approximations, but at the same time generates a lot of small clusters around them and also generates some noisy clusters. Clearly, PL-RGC behaves better than DBScan for this kind of data sets.

\subsection{Chameleon data sets}

Tests were conducted over three different Chameleon benchmark data sets, see Fig. 9. We set the minimum micro-cluster size to 10 , and we check the disjoint-set structure (looking for micro-clusters) every 20 iterations.

Due to the lack of space, we show results for only the ninecluster data set. Figure 10 shows the typical clustering result obtained each 200 iterations up to the end of the process (when the stopping criterion is applied). As expected, the behavior is similar to the one observed on the data sets with Gaussian clusters. Interestingly, the majority of data points inside the clusters are seen to be moving towards some kind of cluster cores (see first column), while almost all the noisy points remain still or barely move. In this way, any cluster shape is detected by the PL-RGC algorithm.

Notice that some "overlapping" clusters are generated by the RGC, for example, in row 1219-third column of Fig. 10 where there are few red points defining a cluster inside and few pink points defining another cluster. However, such clusters are merged when using the refinement process, see Fig. 11. For this data set, the RGC generates 149 representative points capturing the structure of the original data set, 

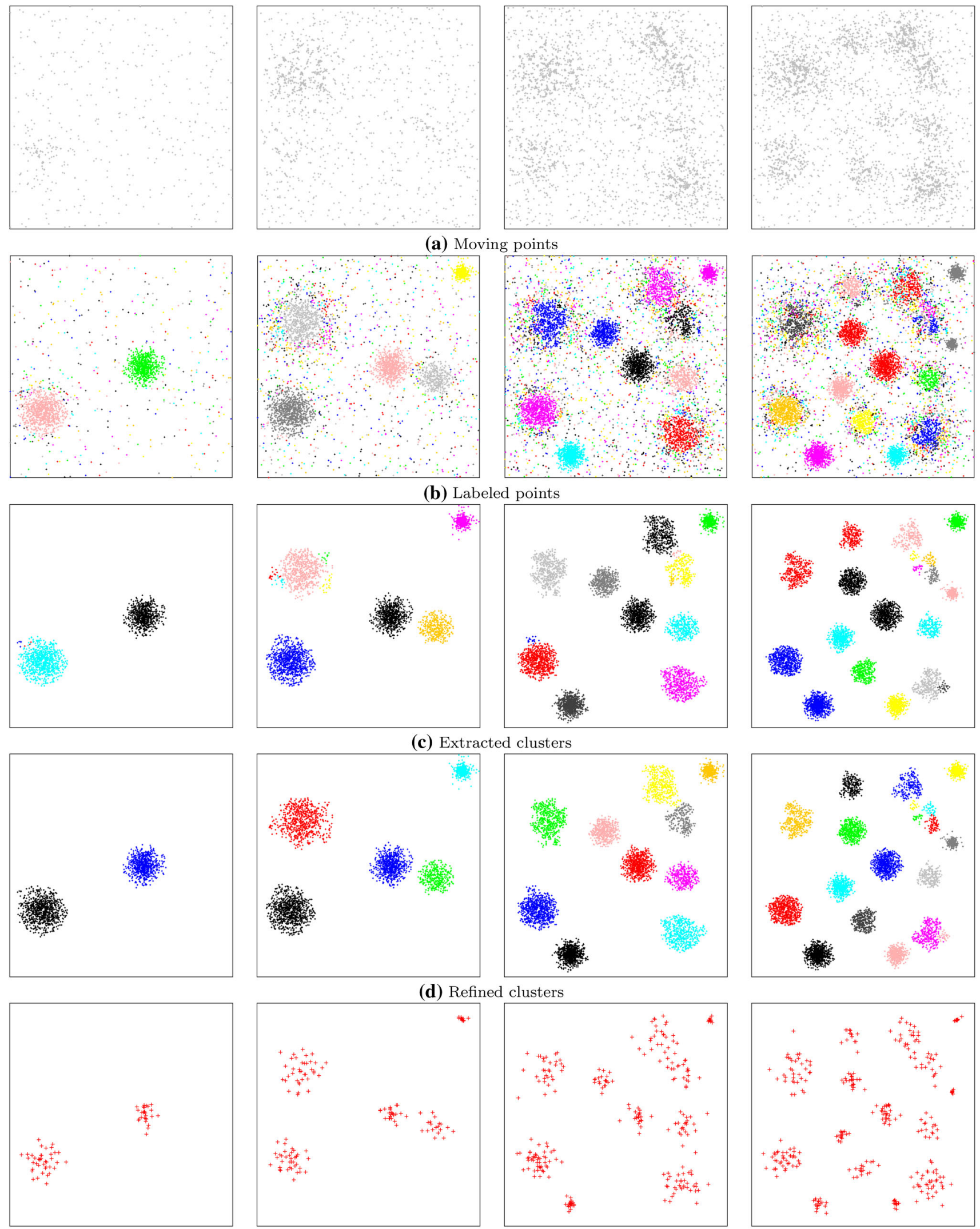

(e) Micro-clusters

Fig. 6 Typical clustering results obtained by the PL-RGC algorithm on the Gaussian data sets 
Epsilon

0.08

0.10

0.12

0.14
6
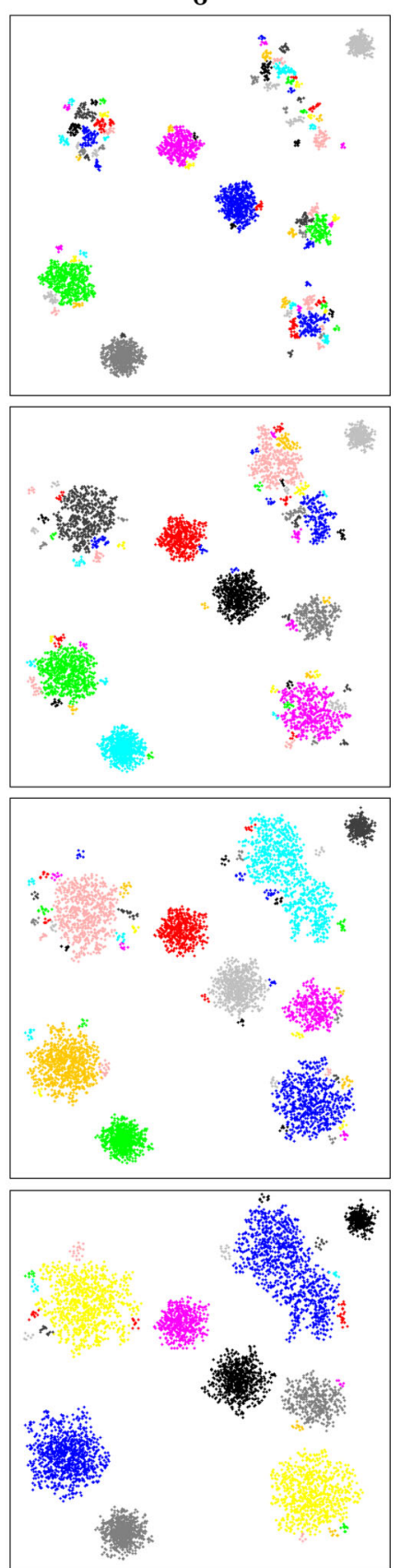

Min points

5
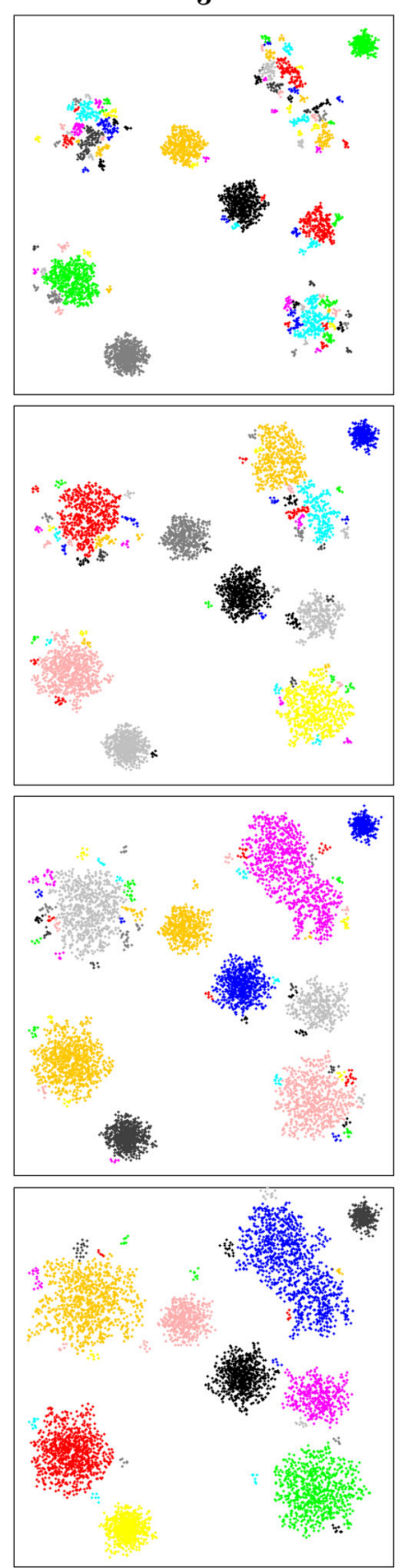
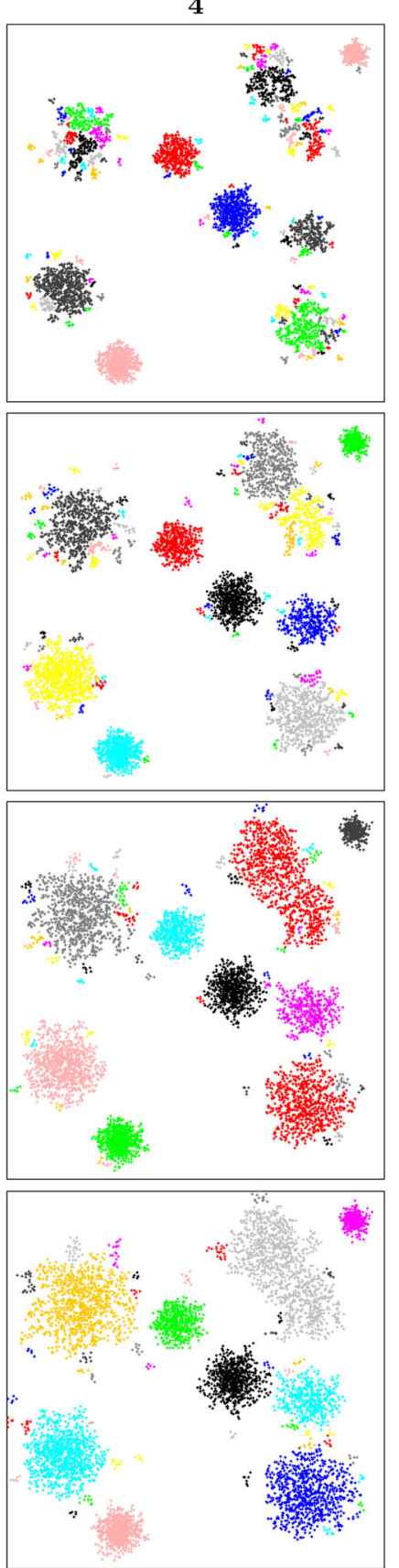

Fig. 7 Results obtained with the DBScan clustering algorithm on the ten Gaussian clusters data set with different parameter values: MinPoints (columns) and Epsilon (rows)

determining appropriately the clusters in the data set and are less than $2 \%$ of the original data set in size (from 8000 data points to 149 representative points). As can be noticed, noisy points are almost totally removed.

Figure 12 shows the behavior of the PL-RGC algorithm on the three Chameleon data sets. As expected, the behavior for all the data sets is similar to that observed on the nine cluster data set. However, for the eight clusters data set, RGC merges three clusters and splits one of them. It can be due to the fact that one of the clusters is not dense and is located very close to a dense cluster. Anyway, all the clusters are extracted, noise is removed and the size of the set of prototypes (micro-clusters) captured is 147 and 167, respectively (close to the $2 \%$ of the data set), similar to the behavior of the nine clusters data set.

We evaluated the performance of PL-RGC against DBScan, as shown in Fig. 13 on the nine-cluster Chameleon data set 

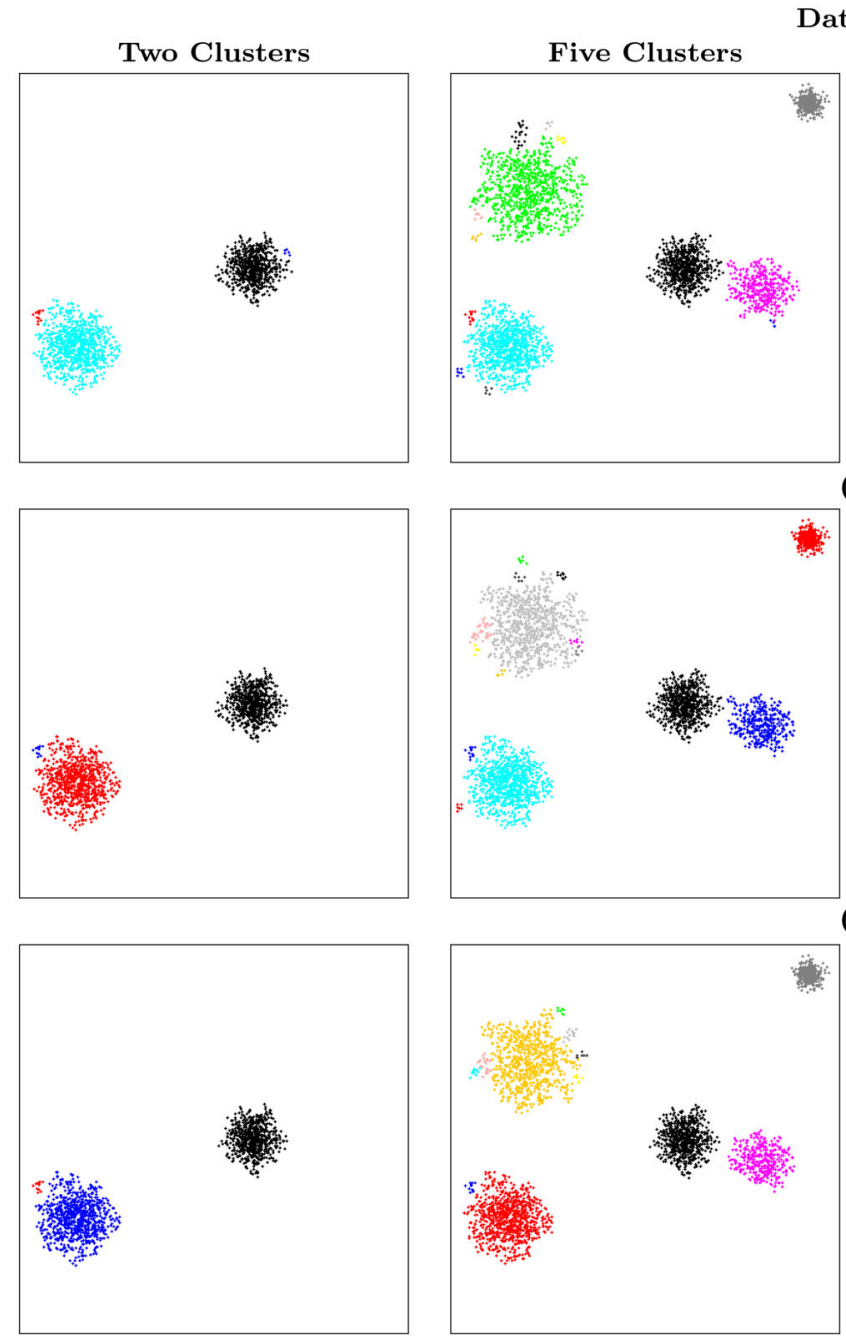

Data Set

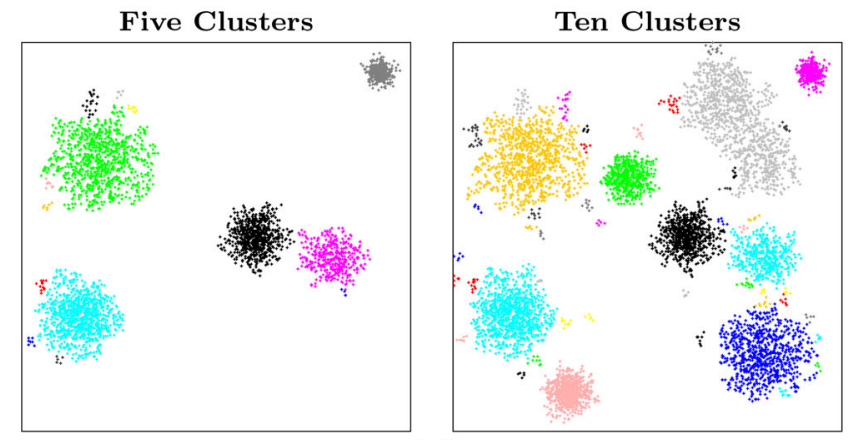

(a)
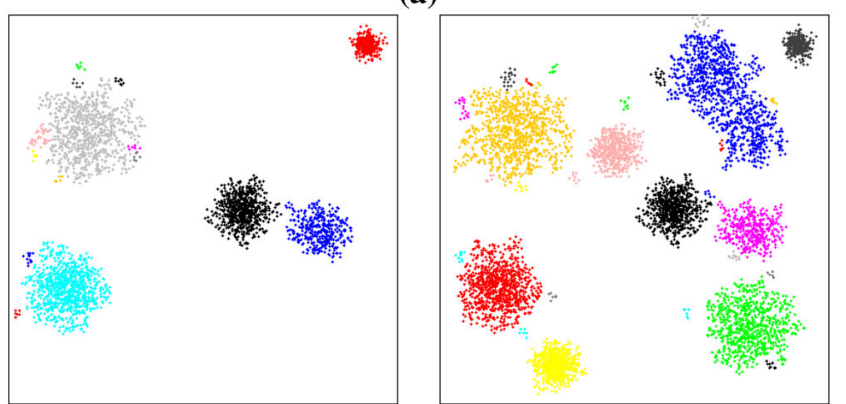

(b)

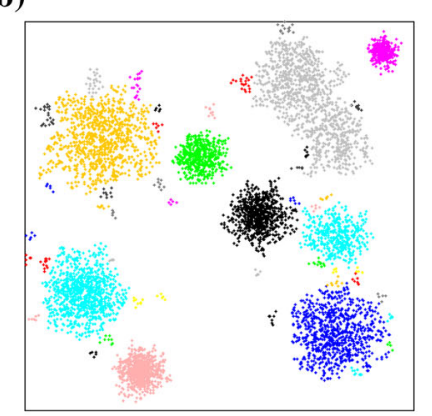

(c)
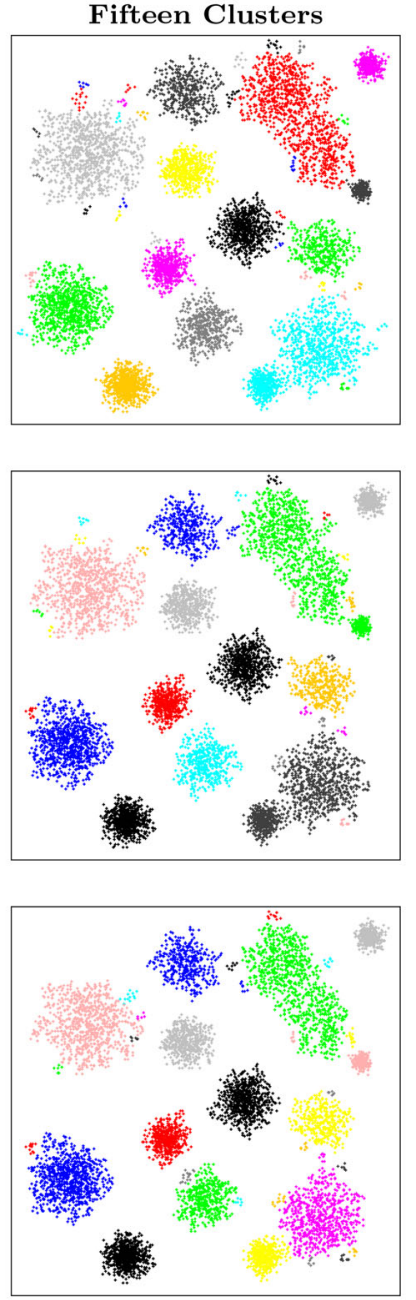

Fig. 8 Results obtained with the DBScan clustering algorithm on the Gaussian clusters data sets with Max Distance of 0.014 and different MinPoints values: a four points, $\mathbf{b}$ five points, and $\mathbf{c}$ six points

Fig. 9 Chameleon data sets: a six clusters, $\mathbf{b}$ nine clusters, and c eight clusters

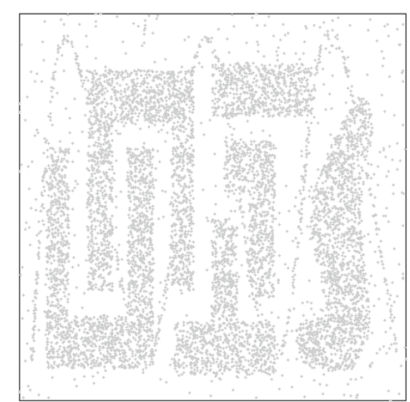

(a)

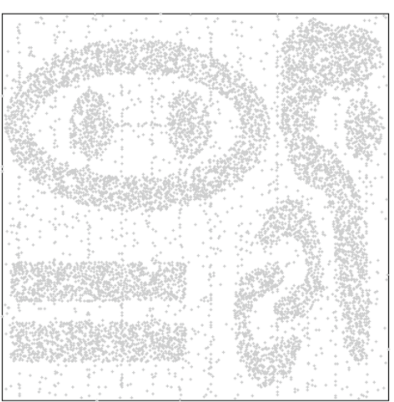

(b)

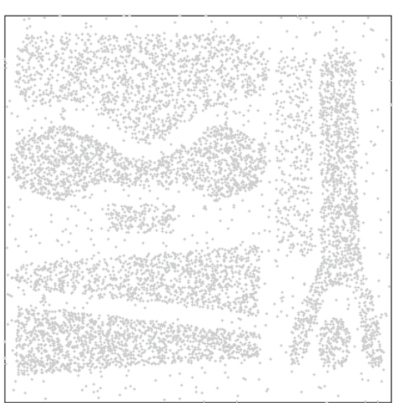

(c) for different values of MinPoints and Epsilon. As can be seen, DBScan is able to find a good approximation of the clusters, if appropriate parameters are given (a similar behavior to the one observed on the Gaussian cluster data sets). Again, we say a good approximation because, in general, DBScan gen- erates several small clusters around the big clusters it finds. Also, some clusters are merged and some noisy clusters are generated.

Figure 14 shows the results of DBScan on the three Chameleon data sets using different MinPoint values, and 


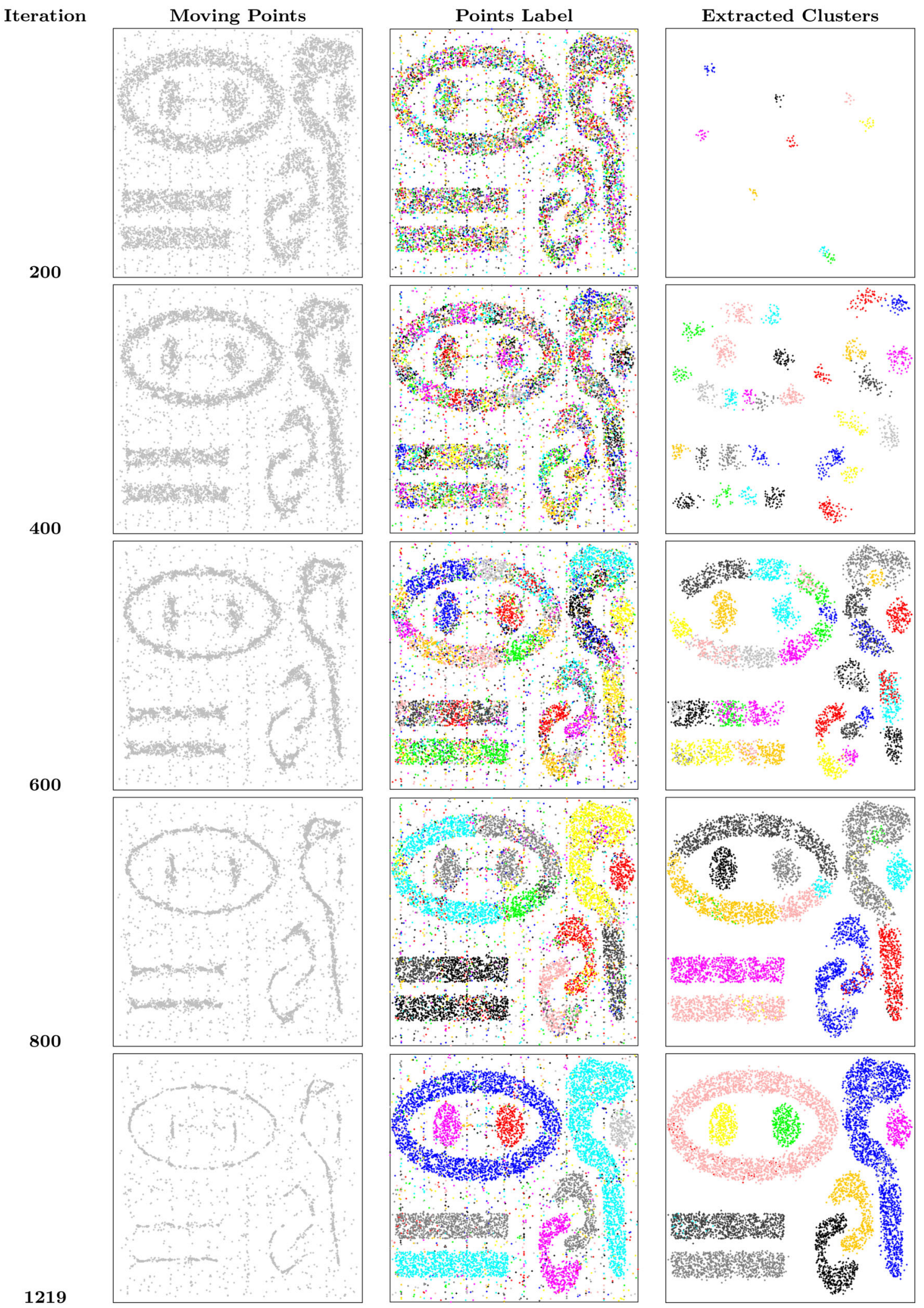

Fig. 10 Typical clustering result for the nine clusters Chameleon data set (t7.10) each 200 iterations. Column one shows the position of the points after the given number of iterations, column two shows the clus- ters obtained after the given number of iterations and column three shows the extracted clusters after such number of iterations 
Fig. 11 Clusters refinement process on the nine clusters Chameleon data set. a Clusters obtained by the PL-RGC algorithm before refinement, and $\mathbf{b}$ clusters obtained by the PL-RGC algorithm after refinement

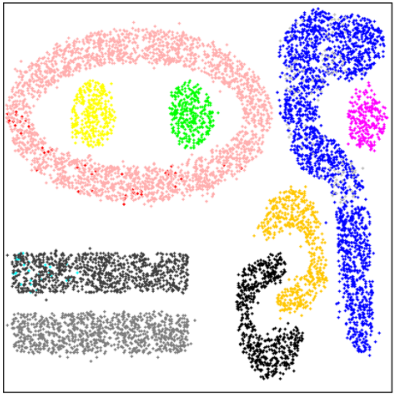

(a)

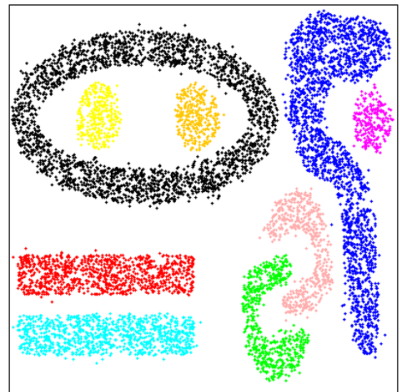

(b)

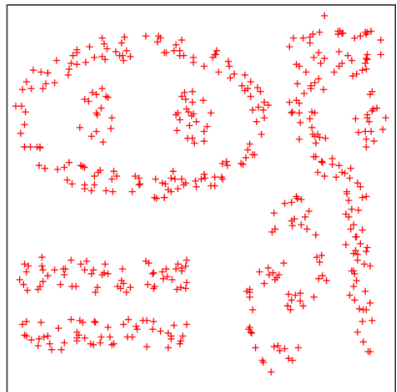

(c)
0.14 as epsilon parameter (the latter fixed to 0.14 based on trial and error). As expected, DBScan finds good cluster approximations, but at the same time generates many small clusters around them in addition to noisy clusters. Clearly, PL-RGC behaves better than DBScan for this kind of data sets.

\section{Experiments with real data}

\subsection{Classic machine learning data sets}

In order to determine the applicability of the proposed approach to real data sets, we used five benchmark data sets (available at [34]), as a testbed, see Table 1.

For these data sets, we used the micro-clusters, generated by the RGC algorithm, as classification model (we labeled each cluster using majority voting). Since the amount of data points is small compared to the amount of points in the previously used data sets, we run the RGC algorithm checking the disjoint-set structure (looking for micro-clusters) each 50 iterations and using three different values of minimum microcluster size $\{2,3,5\}$ to determine an appropriate value for this parameter. Tables 2 and 3 compare the performance reached and the number of micro-clusters generated by the PL-RGC algorithm using these three values.

As expected, the performance reached by the PL-RGC algorithm increases as the minimum numbers of points for defining a micro-clusters decreases (higher granularity). However, the performance reached by PL-RGC is not so affected if setting this value to 5 (the Glass data set is the exception) producing a more compact model for the data set. Table 4 compares the performance reached by the PL-RGC algorithm against the performance reached by the standard $k$ means [2] and the $k$-means++ proposed by Arthur and Vassilvitskii in [35]. As can be noticed, the PL-RGC algorithm usually performs better than $k$-means (only the Glass data set is the exception) and performs fair compared to the $k$-means++.

\subsection{Intrusion detection data}

We use the $10 \%$ of the KDDCup99 data set [34], which is a version of the 1998 DARPA intrusion detection evaluation data set. In this data set, 42 attributes (or fields) that usually characterize network traffic behavior compose each record. Some of these attributes are categorical (8), while others are discrete or numerical (34). The total number of records in the $10 \%$ data set is 49,2021 . This data set contains 22 different types of attacks that can be classified in four main intrusion classes, as is shown in Table 5. The proportion of samples per class is not uniform; for example, from class U2R the number of samples in the training data set is 59; while from class DOS the number of samples is 39,1458 .

This data set was prepared in such a way that only the numerical attributes were used in the training-testing process. Moreover, a numerical attribute was removed since it was the same value for each record in the data set. Therefore, the reduced version of the KDDCup 99 data set contains 33 attributes. Moreover, we created a training data set (removing the class label) with 2,000 normal samples randomly selected from the data set and 2,000 attack samples selected using an stratified sampling strategy. We set the minimum micro-cluster size to 5 , and we check the disjoint-set structure (looking for micro-clusters) each 50 iterations. Table 6 compares the performance reached by the PL-RGC algorithm against the $k$-means algorithm on this data set. Notice that, the PL-RGC algorithm was able to detect three different classes (possibly using some micro-clusters for each of them), while the $k$-means algorithm just recognized two classes (regardless $k$ was set to five). Clearly, the PL-RGC is able to find the internal cluster structure without using the number of clusters of classes in advance. Moreover, the number of microclusters is not so high close to 49 , it represents almost the $1 \%$ of the training data set and close to the $0.001 \%$ of the full data set. Moreover, the false alarm rate is low while maintaining a high attack detection rate. This behavior is desired in network environments.

\section{Conclusions}

We proposed a Parameter-less Randomized Gravitational Clustering algorithm (PL-RGC) by introducing a criterion for stopping the simulation of the gravitational system dynam- 
Fig. 12 Typical clustering results obtained by the PL-RGC algorithm on the Gaussian data sets (last iteration)
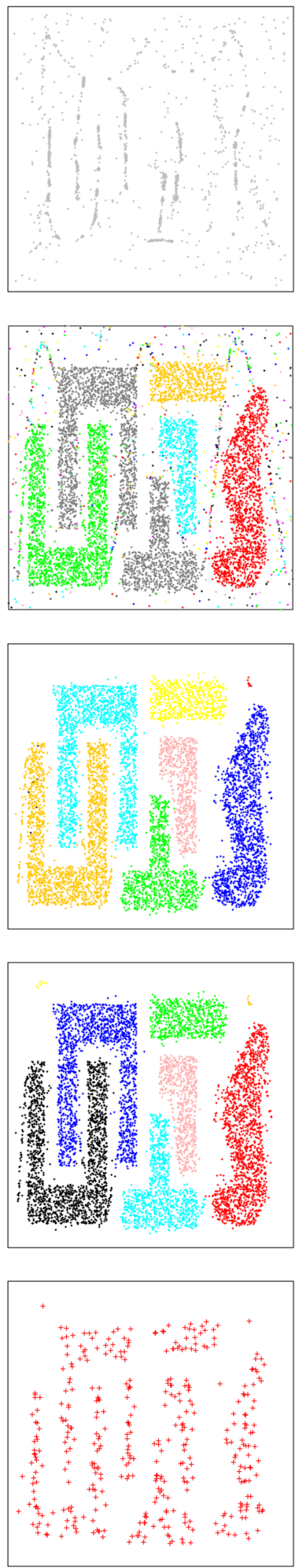

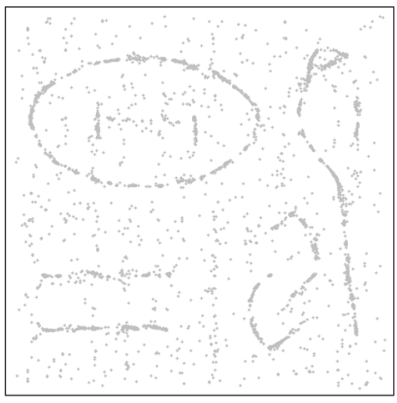

(a) Moving points

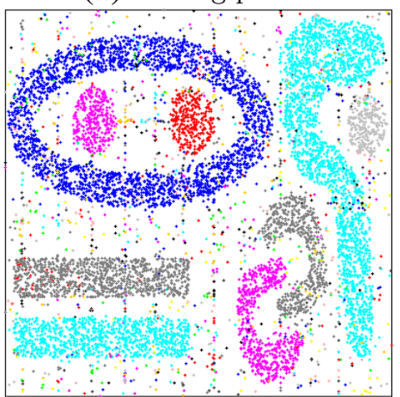

(b) Labeled points

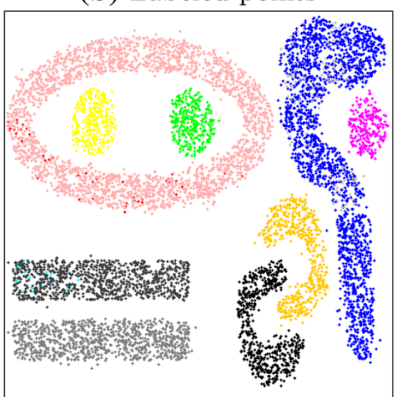

(c) Extracted clusters

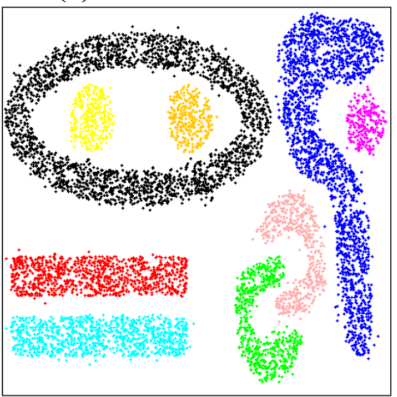

(d) Refined clusters

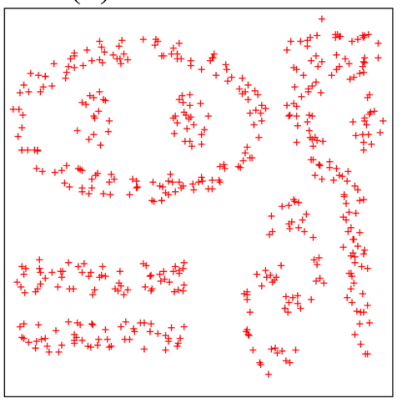

(e) Micro clusters
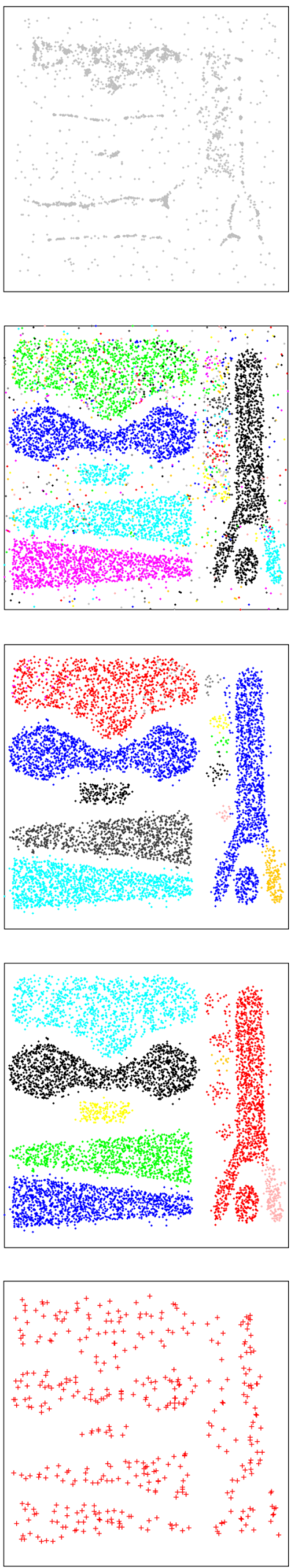

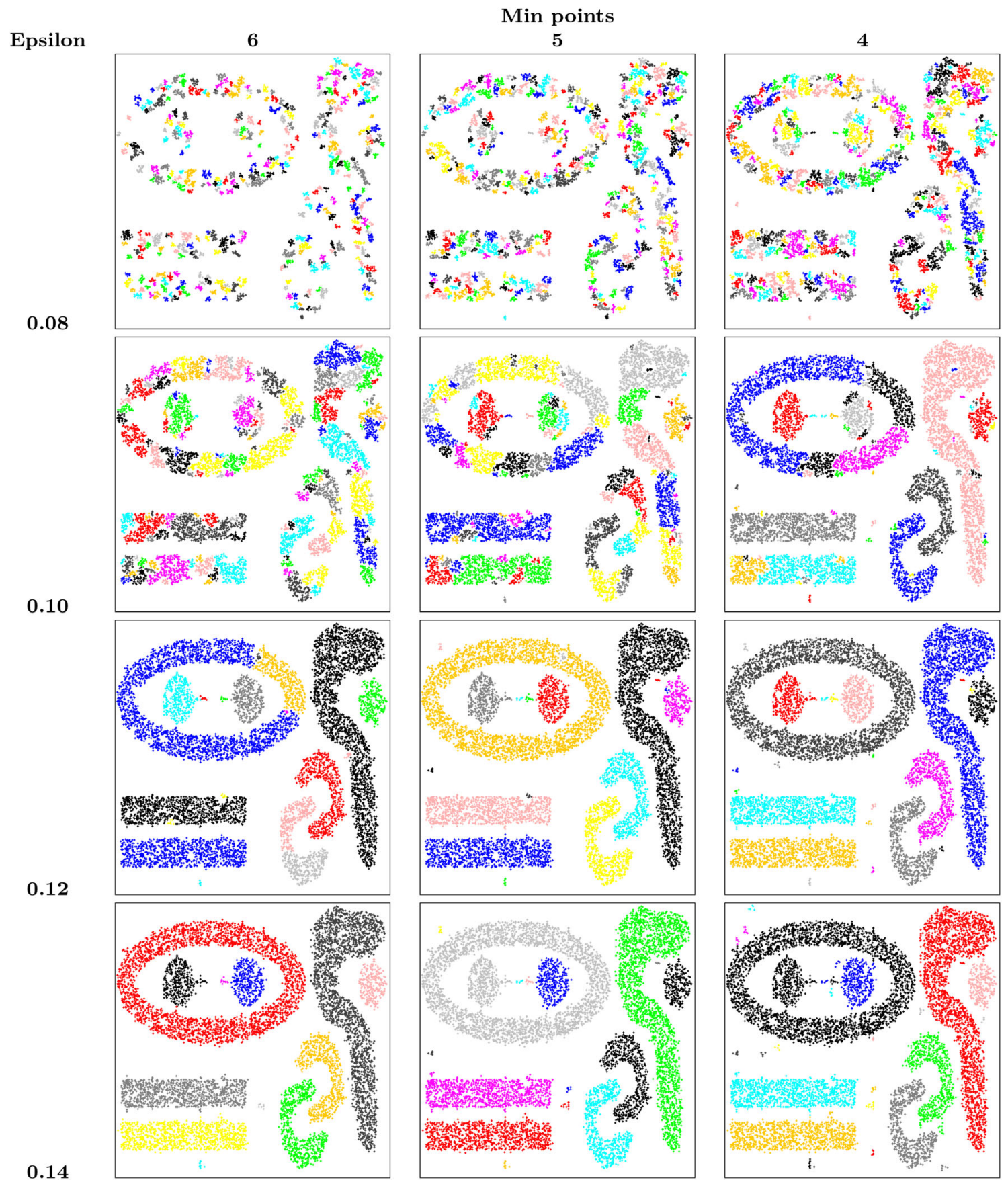

Fig. 13 Results obtained with the DBScan clustering algorithm on the nine-cluster Chameleon data set with different parameter values: MinPoints (columns) and MaxDistance (rows)

ics, based on the average number of points merged iteration by iteration, and by combining the generalization of the RGC algorithm proposed by Gomez et al. in [26], the two extensions of the RGC algorithm proposed by Leon et al. (that uses the rich dynamic of the RGC algorithm and the concept of micro-cluster to represent a cluster as a set of prototypes) in [28] and the one proposed by Gomez et al. (that eliminates the minimum cluster size parameter of the RGC algorithm, and introduced a refinement strategy for merging overlapping clusters) in [27] to obtain a good representation of the internal structure of cluster in a data set using micro-clusters. As shown, the rich dynamic system behavior of the RGC algorithm allows us to get useful information for the clustering process, and introduces several online mechanisms that reduce the parameter setting and improve the quality of the clustering process. The effectiveness of the 
Fig. 14 Results obtained with the DBScan clustering algorithm on the Chameleon data sets with max. distance of 0.014 and different MinPoints values: a four points, $\mathbf{b}$ five points, and $\mathbf{c}$ six points
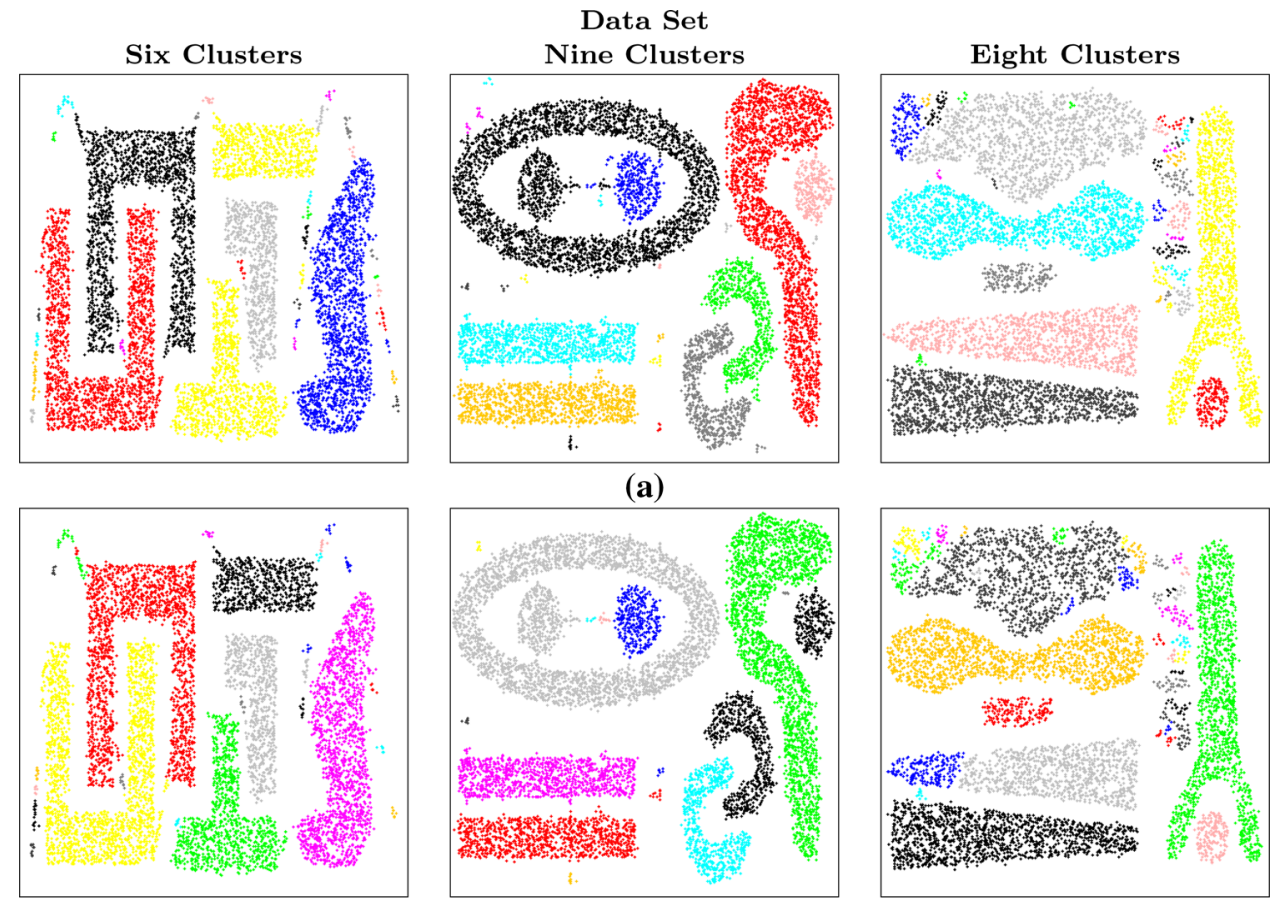

(b)
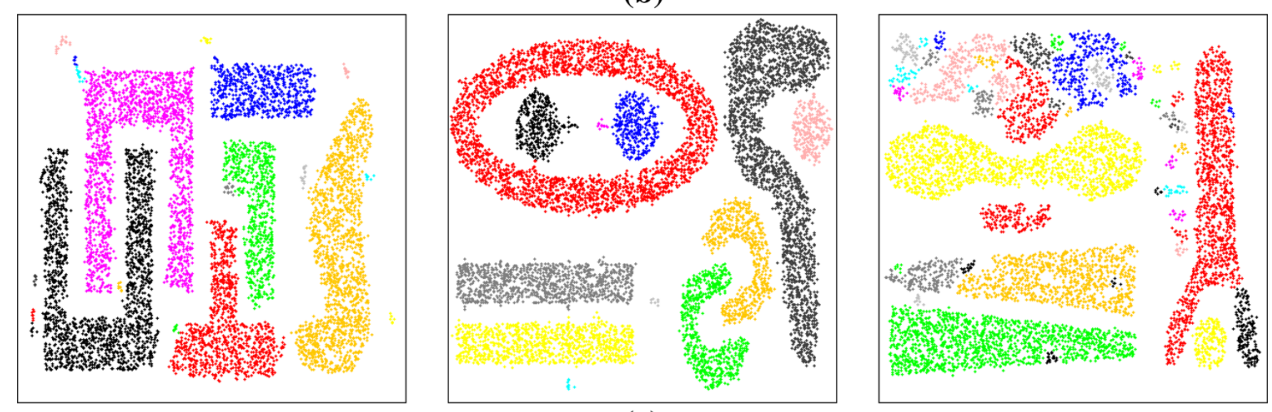

(c)
Table 1 Machine learning testbed

\begin{tabular}{llrll}
\hline Data & M & $n$ & \multicolumn{2}{l}{ Samples } \\
\cline { 3 - 5 } & & & Total & Per class \\
\hline PIMA & 2 & 8 & 768 & $\{500,268\}$ \\
HEART & 2 & 13 & 270 & $\{150,120\}$ \\
IRIS & 3 & 4 & 150 & $\{50,50,50\}$ \\
WINE & 3 & 13 & 178 & $\{59,71,48\}$ \\
GLASS & 6 & 9 & 214 & $\{70,76,17,13,9,29\}$ \\
\hline
\end{tabular}

The second column indicates the number of classes in the data set, the third column is the number of attributes, and the last two columns indicates the total number of instances and instances per class

proposed schemes allows us to extend the original algorithm to be used in data streams [36], since a reduction up to $98 \%$ is obtained from the original data set without losing its structure and dynamic behaviors. This feature is crucial given the constraints of existing memory. Since the PL-RGC algorithm neither requires a special initialization, nor assumes a para-
Table 2 Performance reached by the PL-RGC on the classic machine learning data sets using different values of minimum micro-cluster size

\begin{tabular}{llll}
\hline & 5 & 3 & 2 \\
\hline PIMA & $69.9 \pm 1.6[70.1]$ & $71.6 \pm 0.8[71.9]$ & $73.4 \pm 1.1[73.5]$ \\
HEART & $67.1 \pm 1.8[67.2]$ & $68.0 \pm 1.9[68.0]$ & $69.0 \pm 2.2[69.1]$ \\
IRIS & $87.2 \pm 5.8[90.0]$ & $87.3 \pm 5.6[90.0]$ & $85.4 \pm 7.2[90.0]$ \\
WINE & $73.5 \pm 0.8[73.3]$ & $73.8 \pm 0.8[73.6]$ & $75.7 \pm 1.0[75.8]$ \\
GLASS & $51.1 \pm 3.3[50.5]$ & $53.1 \pm 3.8[51.6]$ & $59.5 \pm 2.4[58.9]$ \\
\hline
\end{tabular}

A value of $a \pm b[c]$ indicates an average performance of $a$ with standard deviation of $b$ and median value of $c$. These values are computed over 30 different runs

metric model, PL-RGC is able to find good clusters, without knowing the number of clusters in advance, regardless of their arbitrary shape, and in the presence of noise. In this direction, our future work will concentrate on exploring and understanding the dynamic system behavior, in such a way, that we can extend the PL-RGC to non-Euclidean spaces, to 
Table 3 Number of micro-clusters generated by the RGC on the classic machine learning data sets using different values of minimum microcluster size

\begin{tabular}{llll}
\hline & 5 & 3 & 2 \\
\hline PIMA & $18.9 \pm 3.0[19]$ & $42.1 \pm 5.2[41]$ & $99.9 \pm 10.5[100]$ \\
HEART & $11.0 \pm 1.9[11]$ & $20.5 \pm 4.7[19]$ & $40.1 \pm 8.3[39]$ \\
IRIS & $5.5 \pm 1.4[6]$ & $9.8 \pm 3.5[10]$ & $14.2 \pm 6.5[12]$ \\
WINE & $12.5 \pm 1.7[13]$ & $20.7 \pm 3.6[21]$ & $38.5 \pm 5.4[40]$ \\
GLASS & $5.1 \pm 0.9[5]$ & $12.4 \pm 1.4[12]$ & $24.3 \pm 2.2[24]$ \\
\hline
\end{tabular}

A value of $a \pm b[c]$ indicates an average number of micro-cluster of $a$ with standard deviation of $b$ and median value of $c$. These values are computed over 30 different runs

Table 4 Comparative performance of $\mathrm{RGC}$ on the classic machine learning data sets

\begin{tabular}{llll}
\hline & $k$-means & $k$-means++ & RGC \\
\hline PIMA & $66.0 \pm 0.0[66.0]$ & $66.8 \pm 0.0[66.8]$ & $69.9 \pm 1.6[70.1]$ \\
HEART & $59.0 \pm 0.2[58.9]$ & $72.9 \pm 9.7[78.9]$ & $67.1 \pm 1.8[67.2]$ \\
IRIS & $85.3 \pm 8.5[88.7]$ & $83.2 \pm 11.8[88.0]$ & $87.2 \pm 5.8[90.0]$ \\
WINE & $69.9 \pm 0.7[70.2]$ & $94.7 \pm 0.5[94.9]$ & $73.5 \pm 0.8[73.3]$ \\
GLASS & $57.0 \pm 3.4[56.5]$ & $42.8 \pm 2.7[42.8]$ & $51.1 \pm 3.3[50.5]$ \\
\hline
\end{tabular}

A value of $a \pm b[c]$ indicates an average performance of $a$ with standard deviation of $b$ and median value of $c$. These values are computed over 30 different runs

Table 5 Classes in $10 \%$ of the KDDCup 99

\begin{tabular}{lrc}
\hline Class & Samples & $\%$ \\
\hline Normal & 95278 & 19.3 \\
U2R & 59 & 0.01 \\
R2L & 1119 & 0.23 \\
DOS & 391458 & 79.5 \\
PRB & 4107 & 0.83
\end{tabular}

User to root attacks (U2R) are buffer_overflow, loadmodule, multihop, perl, and rootkit. Remote to local attacks (R2L) are ftp_write, guess_passwd, imap, phf, spy, warezclient and warezmaster. Denial of service attacks (DOS) are back, land, neptune, pod, smurf and teardrop. Probe attacks (PRB) are ipsweep, nmap, portsweep and satan

Table 6 Comparative performance of RGC on the $10 \%$ KDDCup data set

\begin{tabular}{lll}
\hline & $k$-means & RGC \\
\hline Accuracy & $96.51 \pm 2.66[97.27]$ & $97.99 \pm 0.92[98.22]$ \\
False alarm & $9.86 \pm 14.70[5.59]$ & $4.89 \pm 5.36[3.32]$ \\
Detection rate & $98.65 \pm 0.23[98.57]$ & $99.04 \pm 0.14[99.02]$ \\
Clusters & 5 & $48.6 \pm 1.96[49]$ \\
Recognized classes & 2 & 3 \\
\hline
\end{tabular}

A value of $a \pm b[c]$ indicates an average performance of $a$ with standard deviation of $b$ and median value of $c$. These values are computed over ten different runs deal with the curse of dimensionality when moving to highdimensional spaces, and making it work for data streams. In this last direction, we will use the online strategy for generating prototypes as summarization process of data stream windows, and we will define some mechanisms for merging micro-clusters (cluster fusion), merging clusters with new data points (cluster expansion), merging only new data points (cluster formation) and forgetting factor (cluster extinction). Moreover, we will formally study the gravitational system dynamics to determine, for example, when a cluster is dense and can be recognized by the PL-RGC algorithm, and the minimum micro-cluster size.

\section{References}

1. Jain, A.K.: Data clustering: 50 years beyond $k$-means. Pattern Recognit. Lett. 31(8), 651-666 (2010)

2. MacQueen, J.: Some methods for classification and analysis of multivariate observations. In: Proceeding of the Fifth Berkeley Symposium on Mathematics, Statistics, and Probabilities, vol. 5, pp. 281-297 (1967)

3. Bezdek, J.C.: Pattern Recognition with Fuzzy Objective Function Algorithms. Plenun Press, New York (1981)

4. Jolion, J.M., Meer, P., Bataouche, S.: Robust clustering with applications in computer vision. IEEE Trans. Pattern Anal. Mach. Intell. 13(8), 791-802 (1991)

5. Frigui, H., Krishnapuram, R.: Clustering by competitive agglomeration. Pattern Recognit. 30(7), 1109-1119 (1997)

6. Nasraoui, O., Krishnapuram, R.: A novel approach to unsupervised robust clustering using genetic niching. In: Proceedings of the Ninth IEEE International Conference on Fuzzy Systems, pp. 170-175 (2000)

7. Ester, M., Kriegel, H., Sander, J., Xu, X.: A density-based algorithm for discovering clusters in large spatial databases with noise. In: Proceedings of the Second International Conference on Knowledege Discovery and Data Mining-KDD96, pp. 226-231 (1996)

8. Karypis, E.H.G., Kumar, V.: Chameleon: a hierarchical clustering algorithm using dynamic model. IEEE Comput 32(8), 68-75 (1999)

9. Hinneburg, A., Keim, D.A.: An efficient approach to clustering in large multimedia databases with noise. In: Knowledge Discovery and Data Mining, pp. 58-65 (1998)

10. Nasraoui, O., Krishnapuram, R.: A robust estimator based on density and scale optimization, and its application to clustering. In: Proceedings of the Fifth IEEE International Conference on Fuzzy Systems, pp. 1031-1035 (1996)

11. Jain, A.K., Dubes, R.C.: Algorithms for Clustering Data. Prentice Hall, Englewood Cliffs (1988)

12. Kantardzic, M.: Data Mining: Concepts, Models, and Algorithms. Wiley Interscience, New York (2003)

13. Han, J., Kamber, M.: Data Mining: Concepts and Techniques. Morgan Kaufmann, Burlington (2000)

14. Zhao, Y., Karypis, G.: Comparison of agglomerative and partitional document clustering algorithms. In: Technical Report TR 02-014. Department of Computer Science and Engineering, University of Minnesota (2002)

15. Guha, S., Rastogi, R., Shim, K.: Cure: An efficient clustering algorithm for large data databases. In: Proceedings of the ACM SIGMOD conference on Management of Data, (Seattle Washington), pp. 73-84 (1998)

16. Zhang, T., Ramakrishnan, R., Livny, M.: Birch: An efficient data clustering method for large databases. In: ACM SIGMOD Interna- 
tional Conference on Management of Data, (New York, NY), pp. 103-114. ACM Press, New York (1996)

17. Rousseeuw, P.J., Leroy, A.M.: Robust Regression and Outlier Detection. Wiley, New York (1987)

18. Hampel, F.R., Ronchetti, E.M., Rousseeuw, P.J., Stahel, W.A.: Robust Statistics: The Approach Based on Influence Functions. Wiley, New York (1986)

19. Krishnapuram, R., Keller, J.M.: A possibilistic approach to clustering. IEEE Trans. Fuzzy Syst. 1(2), 98-110 (1993)

20. Beni, G., Liu, X.: A least biased fuzzy clustering method. IEEE Trans. Pattern Anal. Mach. Intell. 16(9), 954-960 (1994)

21. Frigui, H., Krishnapuram, R.: A robust clustering algorithm based on the m-estimator. In: Proceedings of the First International Conference on Neural, Parallel and Scientific Computations, vol. 1, pp. 163-166 (1995)

22. Leon, E., Nasraoui, O., Gomez, J.: A scalable evolutionary clustering algorithm with self-adaptive genetic operators. In: IEEE Conference on Evolutionary Computation (CEC), World Congress on Computational Intelligence, pp. 4010-4017 (2010)

23. Wright, W.E.: Gravitational clustering. Pattern Recognit. 9, 151166 (1977)

24. Kundu, S.: Gravitational clustering: a new approach based on the spatial distri-bution of the points. Pattern Recognit. 32, 1149-1160 (1999)

25. Gomez, J., Dasgupta, D., Nasraoui, O.: A new gravitational clustering algorithm. In: Proceedings of the Third SIAM International Conference on Data Mining 2003, pp. 83-94 (2003)

26. Gomez, J., Nasraoui, O., Leon, E.: Rain: data clustering using randomized interactions between data points. In: Proceedings of the Third International Conference on Machine Learning and Applications (ICMLA 2004), pp. 250-255 (2004)
27. Gomez, J., Leon, E., Nasraoui, O.: Minimum cluster size estimation and cluster refinement for the randomized gravitational clustering algorithm. In: Advances in Artificial Intelligence-IBERAMIA 2012. Lecture Notes in Computer Science, vol. 7637, pp. 51-60 (2012)

28. Leon, E., Gomez, J., Giraldo, F.: Online cluster prototype generation for the gravitational clustering algorithm. In: Advances in Artificial Intelligence-IBERAMIA 2012. Lecture Notes in Computer Science, vol. 7637, pp. 61-70 (2012)

29. Serway, R.A., Jewett, J.W.: Physics for Scientists and Engineers, with Modern Hysics. Thomson-Brooks/Cole, Toronto (2004)

30. Hawking, S.W., Israel, W.: Three Hundred Years of Gravitation. Cambridge University Press, Cambridge (1989)

31. Cormer, T., Leiserson, C., Rivest, R.: Introduction to Algorithms. McGraw Hill, New York (1990)

32. Croft, H.T., Falconer, K.J., Guy, R.K.: Unsolved Problems in Geometry. Springer, New York (1991)

33. Steinhaus, H.: Mathematical Snapshots, 3rd edn. Dover, New York (1999)

34. Bache, K., Lichman, M.: UCI machine learning repository. University of California, School of Information and Computer Science, Irvine, CA (2013). http://archive.ics.uci.edu/ml

35. Arthur, D., Vassilvitskii, S.: $k$-means++: the advantages of careful seeding. In: Proceedings of the eighteenth annual ACM-SIAM symposium on Discrete algorithms, SODA '07, (Philadelphia, PA, USA), pp. 1027-1035. Society for Industrial and Applied Mathematics (2007)

36. Lee, W., Stolfo, S., Mok, K.: Mining in a data-flow environment: experience in network intrusion detection. In: Fifth International Conference on KDD-99, pp. 114-124 (1999) 